\title{
Time-dependent neutrino emission from Mrk 421 during flares and predictions for IceCube
}

\author{
Maria Petropoulou ${ }^{\mathrm{a}, *}$, Stefan Coenders ${ }^{\mathrm{b}, * *}$, Stavros Dimitrakoudis ${ }^{\mathrm{c}}$ \\ ${ }^{a}$ Department of Physics and Astronomy, Purdue University, 525 Northwestern Avenue, West Lafayette, IN 47907, USA \\ ${ }^{b}$ Technische Universität München, Boltzmannstr. 2 (Universecluster), D-85748 Garching bei München, Germany \\ ${ }^{c}$ Department of Physics, University of Alberta, Edmonton, Alberta T6G 2E1, Canada
}

\begin{abstract}
Blazars, a subclass of active galactic nuclei, are prime candidate sources for the high energy neutrinos recently detected by IceCube. Being one of the brightest sources in the extragalactic X-ray and $\gamma$-ray sky as well as one of the nearest blazars to Earth, Mrk 421 is an excellent source for testing the scenario of the blazar-neutrino connection, especially during flares where time-dependent neutrino searches may have a higher detection probability. Here, we model the spectral energy distribution of Mrk 421 during a 13day flare in 2010 with unprecedented multi-wavelength coverage, and calculate the respective neutrino flux. We find a correlation between the $>1 \mathrm{PeV}$ neutrino and photon fluxes, in all energy bands. Using typical IceCube through-going muon event samples with good angular resolution and high statistics, we derive the mean event rate above $100 \mathrm{TeV}(\sim 0.57 \mathrm{evt} / \mathrm{yr})$ and show that it is comparable to that expected from a four-month quiescent period in 2009. Due to the short duration of the flare, an accumulation of similar flares over several years would be necessary to produce a meaningful signal for IceCube. To better assess this, we apply the correlation between the neutrino and $\gamma$-ray fluxes to the $6.9 \mathrm{yr}$ Fermi-LAT light curve of Mrk 421. We find that the mean event count above $1 \mathrm{PeV}$ for the full IceCube detector livetime is $3.59 \pm 0.60(2.73 \pm 0.38) v_{\mu}+\bar{v}_{\mu}$ with (without) major flares included in our analysis. This estimate exceeds, within the uncertainties, the $95 \%$ (90\%) threshold value for the detection of one or more muon (anti-)neutrinos. Meanwhile, the most conservative scenario, where no correlation of $\gamma$-rays and neutrinos is assumed, predicts $1.60 \pm 0.16 v_{\mu}+\bar{v}_{\mu}$ events. We conclude that a non-detection of high-energy neutrinos by IceCube would probe the neutrino/ $\gamma$-ray flux correlation during major flares or/and the hadronic contribution to the blazar emission.
\end{abstract}

Keywords: astroparticle physics, neutrinos, radiation mechanisms: non-thermal, BL Lacartae objects: individual: Mrk 421

\section{Introduction}

Ground-based imaging Cherenkov observatories, such as H.E.S.S. [1], MAGIC [2] and VERITAS [3], in synergy with 4 the Fermi-Large Area Telescope (LAT) [4], have accumulated sufficient $\gamma$-ray data to convincingly prove that blazars, a class 6 of active galactic nuclei (AGN) whose jets point along our line of sight, are efficient particle accelerators. It is commonly accepted that particle acceleration, which, in principle, affects both electrons and protons, takes place in an "active" region of the blazar jet, such as a standing shockwave $[5,6]$ or in sites of relativistic magnetic reconnection [7-9]. If this is the 12 case, then it is expected that both leptonic and hadronic emission processes will contribute to the production of the multi14 wavelength (MW) blazar emission (for a review, see [10, 11]). In a nutshell, in such scenarios the characteristic blazar spectral 16 energy distribution (SED) that shows two humps in a luminosity vs. frequency diagram $[12,13]$ is explained in terms of elec18 tron synchrotron radiation (from radio up to UV/X-rays) and of

\footnotetext{
${ }^{*}$ Principal corresponding author, Einstein Postdoctoral Fellow

** Corresponding author

Email addresses: mpetropo@purdue . edu (Maria Petropoulou), stefan.coenders@tum.de (Stefan Coenders),dimitrak@ualberta.ca (Stavros Dimitrakoudis)
}

hadronic-related processes (from $\mathrm{MeV}$ to $\mathrm{TeV} \gamma$-rays). The latter include proton synchrotron radiation [14-16], pion-related cascades $[17,18]$ and synchrotron radiation of pion-produced pairs $[19,20]$.

Although theoretical models invoking high-energy protons have similar success to leptonic models in fitting the SEDs of blazars [20-25], there is still no direct evidence of proton acceleration in blazar jets (for searches of correlation between AGN and ultra-high energy cosmic-ray events, see also [26-30]). The ultimate proof for the existence of high-energy protons in blazar jets can come only from the detection of high-energy neutrinos [e.g. 31, 32].

Neutrino production in AGN flares has been modeled in anticipation of observations by previous neutrino telescopes, such as AMANDA [33]. It was also postulated by [34] that electron neutrinos produced during AGN flares could be observable by Fly's Eye, a cosmic-ray observatory. Both those models focused on flat spectrum radio quasars (FSRQ), and in particular $3 \mathrm{C} 279$, as they were assumed to have higher neutrino luminosities than BL Lacs. The inherent difficulty in modeling "orphan" $\mathrm{TeV}$ flares (i.e. with no X-ray counterparts) with leptonic synchrotron self-Compton (SSC) emission [35] made such events an enticing target for hadronic models; particularly the 2002 flare of 1ES 1959+650, which was investigated in that regard 
by Halzen and Hooper [36] and Reimer et al. [37]. Soon after${ }_{44}$ wards, Dermer et al. [38] presented a more detailed analytical calculation of expected neutrino emission during FSRQ flares, taking photon-photon $(\gamma \gamma)$ absorption into account.

An accurate modeling of the neutrino emission in both qui48 escent and flaring states of blazar emission, which acts complementary to model-independent studies [e.g. 32, 39-41], is vital

50 for the interpretation of observations by neutrino telescopes, especially in the context of the recent discovery of astrophysical $[42,43]$. In fact, the neutrino spectrum extends to the multi-

${ }_{54} \mathrm{PeV}$ energy range thanks to the newest IceCube detection of a track-like neutrino event with energy significantly above $2 \mathrm{PeV}$

56 [44]. The neutrino flux expected from a non-flaring blazar in the context of a specific leptohadronic model for the blazar SED 58 was recently presented in [19]-henceforth, DPM14, where the blazar Mrk 421 was used as a testbed. This is one of the near-

60 est $(z=0.031,[45])$ and brightest BL Lac sources in the very high energy (VHE; $E_{\gamma}>200 \mathrm{GeV}$ ) sky [e.g. 46] and extra62 galactic X-ray sky, which makes it an ideal target of MW observing campaigns. In particular, the results of the $2009 \mathrm{MW}$ campaign [47], which covers approximately a four month nonflaring period ("quiescence") of Mrk 421 were used in DPM14.

66 The compiled time-averaged SED was modeled using a numerical leptohadronic code [48] that self-consistently treats the energy losses of all radiating particles in the active region of the blazar. Implications of our model regarding other individual blazars and the neutrino background emission from the whole BL Lac population were presented, respectively, in [25] and [49].

In this paper, we expand upon the work of DPM14 by studying the neutrino emission from Mrk 421 during a flaring period in both X- and $\gamma$-ray energy bands. To this end, we apply our model to the 13-day flare of 2010 (MJD 55265-55277), having an unprecedented MW (from radio up to $\mathrm{TeV} \gamma$-rays) and simultaneous (within 2-3 hours) coverage [50]. This dataset, with its wide coverage in energy and time domains, offers a unique opportunity to:

- test the applicability of the model to an active state of blazar emission;

- study the evolution of the neutrino spectrum during a period of flaring activity and calculate the respective neutrino light curve;

- test possible correlations between the neutrino and photon fluxes in different energy bands (e.g. X-rays and $\gamma$ rays);

- calculate the neutrino flux from Mrk 421 during a $\gamma$ ray flare and compare it against the one expected from a longer, but non-flaring period, i.e. in quiescence;

- make predictions about the cumulative number of neutrino events that IceCube should detect in $t$ years, after applying the photon-neutrino flux correlations, if any, to the long-term $\gamma$-ray (Fermi-LAT) light curve of Mrk 421.
By investigating the aforementioned issues, we plan to address the more general question of whether $\gamma$-ray flares determine the optimum time window for high-energy neutrino detection from the nearby blazar Mrk 421.

This paper is structured as follows. In $\$ 2$ we outline the adopted theoretical framework and the numerical code. A description of the IceCube technical characteristics that enter the neutrino event rate calculation are presented in $\S 3$. The results of our model application to the flaring period of Mrk 421 in March 2010 are presented in $\S 4$. We estimate the cumulative number of neutrino events from Mrk 421 in the five years of full IceCube livetime in $\$ 5$, proceed in $\$ 6$ with a discussion of our results and conclude in $\$ 7$.

For the calculation of the expected number of events by IceCube, we will focus on searches that use up-going muons [51-53] rather than high-energy starting events (HESE) [43, 54]. Thanks to a better reconstruction accuracy, larger statistics and lower energy thresholds, the up-going muon samples are better suited to searching for faint signals from potential neutrino point sources, as we will discuss in more detail in \$3. For the required transformations between the reference systems of the blazar and the observer, we have adopted a cosmology with $\Omega_{\mathrm{m}}=0.3, \Omega_{\Lambda}=0.7$ and $H_{0}=70 \mathrm{~km} \mathrm{~s}^{-1}$ $\mathrm{Mpc}^{-1}$. The redshift of Mrk $421 z=0.031$ corresponds to a luminosity distance $d_{\mathrm{L}}=136 \mathrm{Mpc}$.

\section{The model}

\subsection{Theoretical framework}

We adopt a one-zone leptohadronic model for the blazar emission, where the low-energy emission of the blazar SED is attributed to synchrotron radiation of relativistic electrons and the observed high-energy $(\mathrm{GeV}-\mathrm{TeV})$ emission is assumed to have a photohadronic origin.

In particular, we assume that the region responsible for the blazar emission can be described as a spherical blob of radius $R$, containing a tangled magnetic field of strength $B$ and moving with a Doppler factor $\delta$. Protons and (primary) electrons are accelerated by some mechanism whose details lie outside the immediate scope of this work. They are subsequently injected isotropically in the volume of the blob with a constant rate, which is parametrized as $\ell_{\mathrm{i}, \mathrm{inj}}=L_{\mathrm{i}, \mathrm{inj}} \sigma_{\mathrm{T}} / 4 \pi R m_{\mathrm{i}} c^{3}$, where $L_{\mathrm{i}, \text { inj }}$ denotes the injection luminosity as measured in the rest frame of the emitting region, $\sigma_{\mathrm{T}}=6.65 \times 10^{-25} \mathrm{~cm}^{2}$ is the Thomson cross section and the subscript $\mathrm{i}$ denotes protons or electrons $(i=p, e)$. These are assumed to escape from the emitting region in a characteristic timescale, which is set equal to the photon crossing time of the source, i.e. $t_{\mathrm{p}, \text { esc }}=t_{\mathrm{e}, \mathrm{esc}}=R / c$. Their distributions at injection are described as power-laws with index $s_{\mathrm{i}}$ in the energy range $E_{\mathrm{i}, \min }=\gamma_{\mathrm{i}, \min } m_{\mathrm{i}} c^{2}$ to $E_{\mathrm{i}, \max }=\gamma_{\mathrm{i}, \max } m_{\mathrm{i}} c^{2}$.

Photons, neutrons and neutrinos complete the set of the five stable populations, that are at work in the blazar emitting region. Pions $\left(\pi^{ \pm}, \pi^{0}\right)$, muons $\left(\mu^{ \pm}\right)$and kaons $\left(K^{ \pm}, K^{0}\right)$ constitute the unstable particle populations, since they decay into lighter particles. The production of pions is a natural outcome of photohadronic interactions between the relativistic protons and the 
internal photons; the latter are predominantly synchrotron photons emitted by the primary electrons. The decay of charged pions results in the injection of secondary relativistic electrons and positrons $\left(\pi^{ \pm} \rightarrow \mu^{ \pm}+v_{\mu}\left(\bar{v}_{\mu}\right), \mu^{ \pm} \rightarrow e^{ \pm}+\bar{v}_{\mu}\left(v_{\mu}\right)+v_{\mathrm{e}}\left(\bar{v}_{\mathrm{e}}\right)\right)$, whose synchrotron emission emerges in the GeV-TeV regime, for a certain range of parameter values. Neutral pions decay into VHE $\gamma$-rays (e.g. $E_{\gamma} \sim 10 \mathrm{PeV}$, for a parent proton with energy $E_{\mathrm{p}}=100 \mathrm{PeV}$ ), and those are, in turn, susceptible to $\gamma \gamma$ absorption and can initiate an electromagnetic (or hadronic) cascade $[17,55]$. As SSC emission from primary electrons may also emerge in the $\mathrm{GeV}-\mathrm{TeV}$ energy band, the observed $\gamma$-ray emission can be totally or partially explained by photohadronic processes, depending on the specifics of individual sources [25].

Besides neutrinos produced by photohadronic interactions between protons and photons in the emission region of Mrk 421, an additional component (cosmogenic neutrinos) may emerge from the interaction of escaping protons from the source with the background radiation fields, such as the extragalactic background light (EBL) [56]. In this study, we will neglect the cosmogenic neutrino component, for reasons to be discussed in $\S 6$.

\subsection{Numerical framework}

The interplay of the processes governing the evolution of the energy distributions of the five stable particle populations is formulated with a set of five time-dependent, energy-conserving kinetic equations. To simultaneously solve the coupled kinetic equations for all particle types we use the time-dependent code described in [48]. Photopion interactions are modeled using the results of the Monte Carlo event generator sopHIA [57], while Bethe-Heitler pair production is similarly modeled with the Monte Carlo results of Protheroe and Johnson [58] and Mastichiadis et al. [59]. Details of the numerical treatment of shortlived particles (i.e., $\mu^{ \pm}, \pi^{ \pm}, \pi^{0}, K^{ \pm}$and $K^{0}$ ), which are not modeled with kinetic equations, can be found in $[19,60]$. We finally note that for the range of parameter values used in this study, the effect of synchrotron cooling for these unstable particles is negligible.

\section{Neutrino point source detection with IceCube}

The sensitivity of neutrino telescopes to a neutrino point source is limited by vast backgrounds of $\mu^{ \pm}$produced in extensive air showers or by charged-current (CC) interactions of atmospheric $v_{\mu}+\bar{v}_{\mu}$. Still, neutrino telescopes can cope with these backgrounds with focused searches, using track-like events of $\mu^{ \pm}$penetrating the detector $[53,61]$, for the following reasons: (i) the angular reconstruction accuracy reduces the background to a small part of the sky, while the expected mean background rate can be effectively calculated using off-source regions; (ii) track-like events can travel long distances before being detected, thus yielding a large collection volume which increases with energy, yielding an effective area 10-100 times larger than that for starting events [42] (see also Fig. 1); (iii) $\mu^{ \pm}$created in CC $v_{\mu}$ interactions are closely correlated to the parent $v$ direction above $\mathrm{TeV}$ energies (there, the $90 \%$ limit on the direction is well below $1^{\circ}$ ). However, in track-like events, contrary to the cascade events, the information of the $\mu$ and parent $v_{\mu}$ energies is partially lost, since only a small fraction of the energy deposition along the track is observed.

Even though the aforementioned searches are restricted to single-flavor neutrinos $\left(v_{\mu}+\bar{v}_{\mu}\right)$, only muons created in $\mathrm{CC}$ interactions are reconstructed accurately enough to allow for a robust association of a neutrino with an astrophysical point source. Furthermore, the position of Mrk 421 in the northern sky (Dec: $38.19^{\circ}$ ) coincides with that region in the sky where IceCube is most efficient in detecting muon flavored neutrinos (in the energy range of $100 \mathrm{TeV}$ to a couple $\mathrm{PeV}$ ). A small additional component can arise from $v_{\tau}$ $\mathrm{CC}$ interactions followed by the sub-sequent decay of the $\tau$-lepton into $\mu v_{\mu} v_{\tau}$ with branching ratio of $17 \% \%^{1}$. In addition, regeneration of $v_{\tau}$ occurs during propagation within the Earth [62], increasing the flux at lower energies. The effect of $\tau$ neutrinos on our calculated rates is expected to be $\lesssim 10 \%$ (compare [63]), i.e. smaller than other uncertainties considered in this work. Thus, in what follows we consider only the muon component of the neutrino signal.

Taking into account the performance of the completed IceCube detector for up-going track-like events [53], the expected (mean) number of (anti-)neutrinos is then calculated by

$$
N_{v}=T \int_{E_{v, \text { min }}}^{E_{\gamma, \text { max }}} d E_{v} \int_{\Delta \Omega\left(E_{v}\right)} \mathrm{d} \Omega A_{\mathrm{eff}}\left(E_{v}, \vec{x}\right) \sum_{i} \frac{\partial^{2} F_{v, i}}{\partial \Omega \partial E_{v}},
$$

where $E_{v, \text { min }}=100 \mathrm{GeV}, E_{v, \text { max }}=100 \mathrm{PeV}, \partial^{2} F_{v, i} / \partial \Omega \partial E_{v}$ is the incident muon neutrino flux for different flux components $i$, and $\Delta \Omega$ is the observation window around the source position $\vec{x}$. Equation (1) shows that the mean number of events measured by IceCube depends mainly on:

1. the energy range of the flux; neutrino telescopes such as IceCube start to observe neutrinos in point source searches at $\mathrm{TeV}$ energies. At higher energies, the increasing crosssection $\sigma_{\mathrm{CC}}\left(v_{\mu}+X \rightarrow \mu+Y\right)$ enhances the effective area $A_{\text {eff }}$ for neutrinos (see Fig. 1).

2. the point of observation; for the position of Mrk 421 on the sky (Ra: $166.07^{\circ}$, Dec: $38.19^{\circ}$ ), the effective area $A_{\text {eff }}$ of IceCube increases up until $1 \mathrm{PeV}$ before Earth absorption becomes dominant (see Fig. 1).

3 . the contamination of the signal; for the northern sky, atmospheric $\bar{v}_{\mu}+v_{\mu}$ form an irreducible background over the high-energy signal. This will be exemplified later in $\$ 4.2$ for the case of Mrk 421 .

4. the integration time of observation $T$.

In what follows, we assume $90 \%$ of all $v_{\mu}+\bar{v}_{\mu}$ events to be reconstructed within $1^{\circ}$, neglecting the energy dependence of the IceCube median angular resolution, $\Delta \Psi$. Given that resolution, which is shown in Fig. 2, our choice provides a conservative estimate of the event number.

${ }^{1}$ Due to the three-body decay of the $\tau$ lepton, the energy of the final $\mu$ will be lower than that of a $\mu$ produced by $\mathrm{CC}$ interactions of muon neutrinos. 


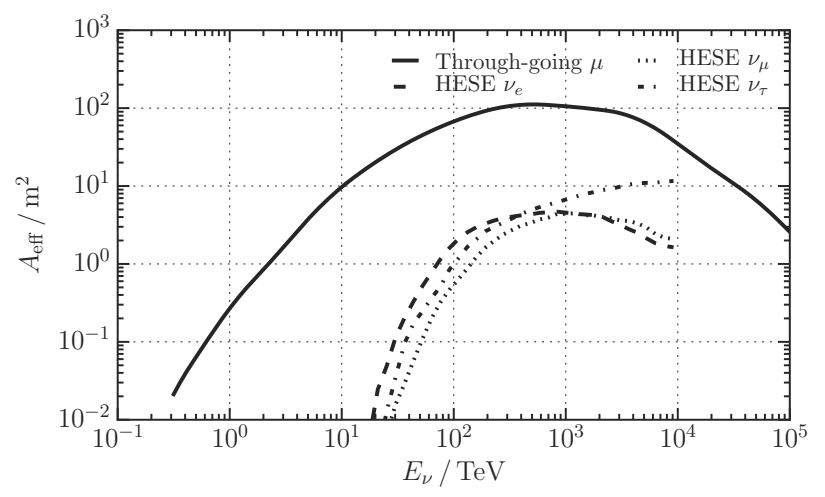

Figure 1: Effective area of IceCube $A_{\text {eff }}$ at the position of Mrk 421 (Ra: $166.07^{\circ}$, Dec: $38.19^{\circ}$ ) with respect to the primary neutrino energy $E_{v}$. The effective area is shown for typical upgoing muon analysis (solid line) and compared to that of the high-energy starting event (HESE) analysis for $v_{\mathrm{e}}$ (dashed line), $v_{\mu}$ (dotted line), and $v_{\tau}$ (dashed-dotted line). Data are adopted from [53, 54].

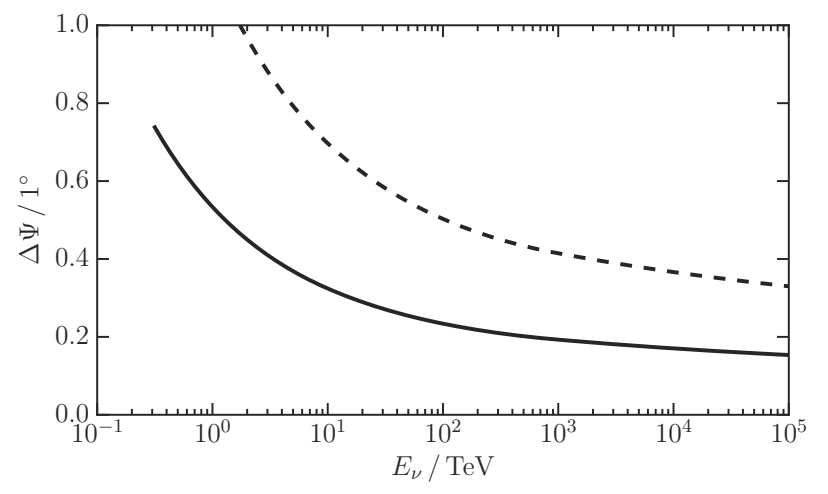

Figure 2: Energy dependence of the median angular resolution of IceCube $\Delta \Psi$ (solid line) and of the estimated $90 \%$ upper limit (dashed line) assuming a Gaussian distribution of the angular uncertainty. Data are adopted from [53].
The incident neutrino flux $\partial^{2} F_{v, i} / \partial \Omega \partial E_{v}$ is primarily composed of three components. The first component is the signal from Mrk 421. Its flux is reduced by $10 \%$ of events that are reconstructed outside of the observation window. The other two components are related to the background neutrino emission which, in turn, consists of (i) the conventional atmospheric flux with a soft spectrum of approximately $\sim E^{-3.7}[64]$ and (ii) the astrophysical flux, as measured by IceCube with a spectral index $\sim E_{v}^{-2.3}$ [43]. Here, we treat this component as purely isotropic, thus, forming an additional background at high energies for searches of point-like neutrino sources. Within the window size $\Delta \Omega$, which is small compared to the variations of reconstruction accuracy and effective area, the neutrino flux and effective area are assumed to be constant. Thus, the integral over the solid angle in eq. (1) reduces to a constant $\Delta \Omega$. An additional prompt neutrino component is neglected, as it is subdominant to the atmospheric or diffuse flux, both in the lowand high-energy regimes, respectively.

\section{The 13-day flare of 2010}

Here, we present our results on the photon and neutrino emission from the blazar Mrk 421 during the 13-day flaring event of 2010, focusing on the expected neutrino event rate from that flare and on the calculation of the IceCube sensitivity for Mrk 421. We then extrapolate our findings using the longterm ( 6.9 yr) Fermi-LAT $\gamma$-ray light curve of Mrk 421 and make predictions about the cumulative number of events that IceCube should detect in the following years of its operation.

The SEDs for the period MJD 55265-55277 were modeled by varying six out of the eleven free model parameters (see Table A.6), while the rest of them were kept fixed to the following values: $B=5 \mathrm{G}, R=3.2 \times 10^{15} \mathrm{~cm}, \gamma_{\mathrm{e}, \min }=100, \gamma_{\mathrm{p}, \min }=1$ and $s_{\mathrm{p}}=1.2$. The values we chose for each of the six varying parameters may not necessarily correspond to the best possible fit for each day, as would be expressed by a $\chi^{2}$ minimum. Nevertheless, a good agreement between the model and the MW data is obtained for the whole duration of the flare. As the neutrino spectra are not sensitive to small changes in the model parameter values, the derived neutrino rates are robust. This is the same approach as the one followed in DPM14 and [25].

To minimize the computing time required for modeling the 13-day flaring activity we approximated the flaring period by a series of 13 steady-state snapshots. The individual daily SEDs were numerically calculated for different values of the six varying parameters. These were used as initial conditions for the numerical calculation of the final steady state of the system (for continuous parameter variations in time, see e.g. [22]). We note that our approximation is valid as long as the typical time for reaching a steady-state is less than the time interval between two successive snapshots (1 day). Indeed, a steady-state in our simulations was typically achieved within $\sim 3 t_{\mathrm{cr}}=3 \times 10^{5} \mathrm{~s}\left(R / 10^{15} \mathrm{~cm}\right)<2 \times 10^{6} \mathrm{~s}(\delta / 20)(\delta T / 1 \mathrm{~d}) . \mathrm{We}$ finally note that for the adopted parameter values the emission region is optically thin to photopion production (see also §6) with implications on the blazar energetics, which have been discussed in [25, 49].
248 


\subsection{Photon emission}

The observed SEDs of Mrk 421 for the first (MJD 55265) and last (MJD 55277) days of the MW campaign are shown in Fig. 3. To facilitate a comparison, the time-averaged SED over the period MJD 54850-54983 [47], a good representation of the blazar quiescent emission, has been included in the plot (grey points). The model-derived photon spectra for the two days 2010 flare and of the 2009 quiescent period are plotted with thick black and grey lines, respectively. The spectra proferent types of lines (for details, see figure caption). The model Appendix A. We note that the VHE $(>200 \mathrm{GeV})$ observations [50] and [47]. In other words, the VHE $\gamma$-ray spectra shown photon spectra take into account only the intrinsic $\gamma \gamma$ absorption. This also explains the presence of the $\pi^{0} \gamma$-ray bump at $\sim 5-10 \mathrm{PeV}$, which otherwise would be attenuated by the EBL.

322 Figures 3 and A. 10 show that the leptohadronic model provides an overall good description of the data for the 13 consecutive 224 days of the flare.

The Fermi-LAT observations at $\sim 400 \mathrm{MeV}$ are the more constraining for our model, since for the adopted parameter values the latter predicts a luminous Bethe-Heitler component hard X-rays to soft $\gamma$-rays (magenta long-dashed lines) [65]. The Bethe-Heitler component, which is explained as synvia the Bethe-Heitler process, is a distinct feature to be conained with current, e.g. IBIS/INTEGRAL [66] and future, e.g. PANGU [67], $\gamma$-ray satellites operating in the 1-100 MeV pected to be highly polarized and, as such, its modeling constiters, such as ASTROGAM ${ }^{2}$ and AdEPT [68]. A comparison of of the 13-day flare gives insight into the interplay of the differprimary leptonic SSC component (blue dotted-line) peaking at ondary pairs. In fact, the observed $\gamma$-ray emission in the range synchrotron radiation from secondary pairs. These are the byproduct of $\pi^{ \pm}, \mu^{ \pm}$decays (gold dashed-dotted lines) and $\gamma \gamma$ absorption (orange short-dashed lines). It is noteworthy that the one, with the SSC component dominating in $\gamma$-rays, simply by at $\sim 0.1-1 \mathrm{TeV}$; this appears as a high-energy bump in the spectra shown with orange short-dashed lines (see Fig. 3). A fraction of the (sub)TeV radiation is, in turn, attenuated leading to the production of the lower-energy bump of the spectrum that is plotted with orange short-dashed lines in Fig. 3. We note that the full width at half maximum (FWHM) of the two bumps is also related to the FWHM of the respective parent $\gamma$-ray spectra. The (unattenuated) flux produced via photomeson processes (gold dashed-dotted lines) is highest at the start of the 13-day flare (left panel in Fig. 3) and decreases towards the end of the flare (right panel in Fig. 3). Since a fraction of the $\gamma$-ray flux is internally attenuated, the gradual $\gamma$-ray flux decrease over the 13-day period will be also reflected in the synchrotron emission of pairs produced by $\gamma \gamma$ absorption. Indeed, the peak flux of the lower energy bump in the synchrotron spectrum of $\gamma \gamma$ pairs (orange short-dashed lines) has decreased since the start of the 13-day flare (see both panels in Fig. 3).

Finally, the proton synchrotron spectrum (light blue dashed double-dotted lines) is the least variable component, in terms of flux, during the 13-day flare. The peak energy of the spectrum has, however, decreased by approximately a factor of 7 between the start and end of the 13-day flare. The proton synchrotron spectrum peaks in hard X-rays, i.e. $5-35 \mathrm{keV}$, and may have a non-negligible contribution to the observed hard X-ray flux in other, even more extreme, flares (see also MJD 55271 in Fig. A.10). A great example is the major MW flare of April 2013 [69-72], where the fractional variability in hard X-rays (3$79 \mathrm{keV}$ ) as measured by NuSTAR was found to be $0.790 \pm 0.001$ [73], in contrast to $0.42 \pm 0.12$ that was measured with BAT (15$50 \mathrm{keV}$ ) during the 13-day flare [50]. Regardless, the detailed modeling of such an extreme flare across the MW spectrum as well as its implications for the current model will be the subject of a subsequent paper.

The parameter values used in modeling the 13 daily SEDs are summarized in Table A.6. Inspection of the table shows that no major variations of the model parameters were required for explaining the SEDs. In all cases, the parameters change by a factor of $\sim 2.5$ at maximum with respect to their timeaveraged values. We find that an anti-correlation between $\gamma_{\mathrm{p}, \max }$ and $\ell_{\mathrm{p}}$ is required to explain the data. This is an outcome of the adopted flat proton spectrum $p<2$; a simultaneous increase of both $\gamma_{\mathrm{p}, \max }$ and $\ell_{\mathrm{p}}$, would lead to larger variations of the $\gamma$ ray flux than what is actually observed. For the adopted parameters, the electron distribution is modified by synchrotron cooling and the peak synchrotron flux is therefore produced by electrons with Lorentz factors equal to the cooling Lorentz factor. This is defined as the Lorentz factor where the synchrotron cooling time scale $6 \pi m_{\mathrm{e}} c / \sigma_{\mathrm{T}} B^{2} \gamma_{\mathrm{e}}$ equals the dynamical one $t_{\mathrm{cr}}$. Thus, changes of $\gamma_{\mathrm{e}, \max }$ alone do not have a direct effect on the peak synchrotron flux, which, in turn, explains the absence of correlation between $\gamma_{\mathrm{e}, \max }$ and $\ell_{\mathrm{e}}$.

\subsection{Neutrino emission}

The daily all-flavor neutrino $(v+\bar{v})$ spectra are presented in Fig. 4, where thick lines are used for displaying the neutrino emission at the beginning and the end of the 13-day flare.

\footnotetext{
${ }^{2} \mathrm{http}: / /$ astrogam.iaps.inaf.it/scientific_instrument.html
} 

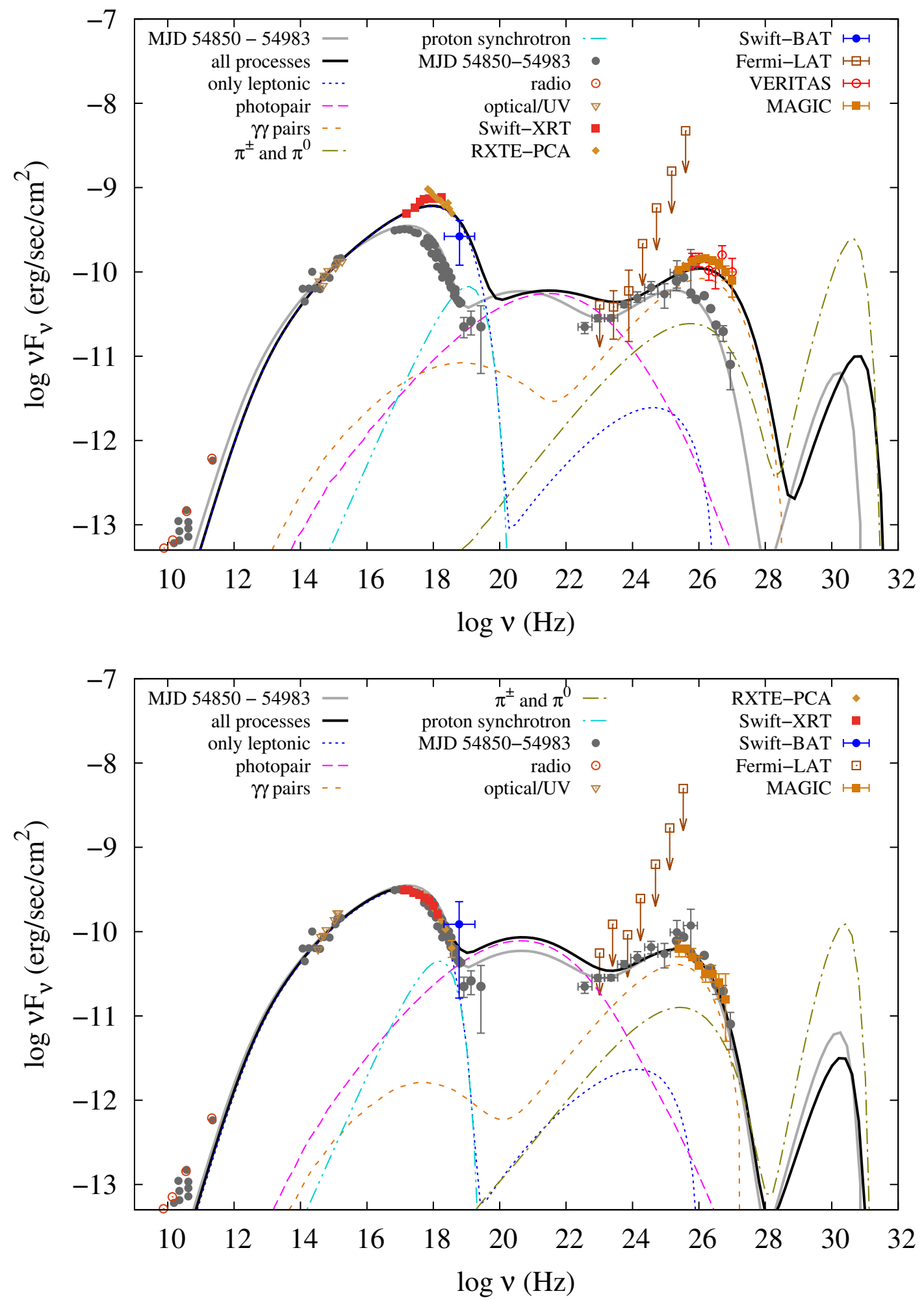

Figure 3: Simultaneous multi-wavelength SED of Mrk 421 on MJD 55265 (top panel) and MJD 55277 (bottom panel). Different symbols denote the various instruments used to collect the data, and their meaning is given in the legends. All data-points are from [50]. The grey circles depict the time-averaged SED of Mrk 421 over the period MJD 54850-54983 [47]. This is a good representation of the blazar non-flaring (quiescent) emission. The model-derived spectra that fit the daily SEDs are plotted with black thick lines. The grey thick lines are a fit to the quiescent emission. Different types of lines are used to present the spectra from different emission processes: proton synchrotron radiation (light blue dashed double-dotted line), (primary) electron synchrotron and SSC emission (blue dotted line), synchrotron radiation from Bethe-Heitler pairs (magenta long-dashed line), synchrotron radiation of pairs from $\pi^{ \pm}, \mu^{ \pm}$decays and $\gamma$-rays from $\pi^{0}$ decays (gold dashed-dotted line), synchrotron radiation of pairs from $\gamma \gamma$ absorption (orange short-dashed line). 


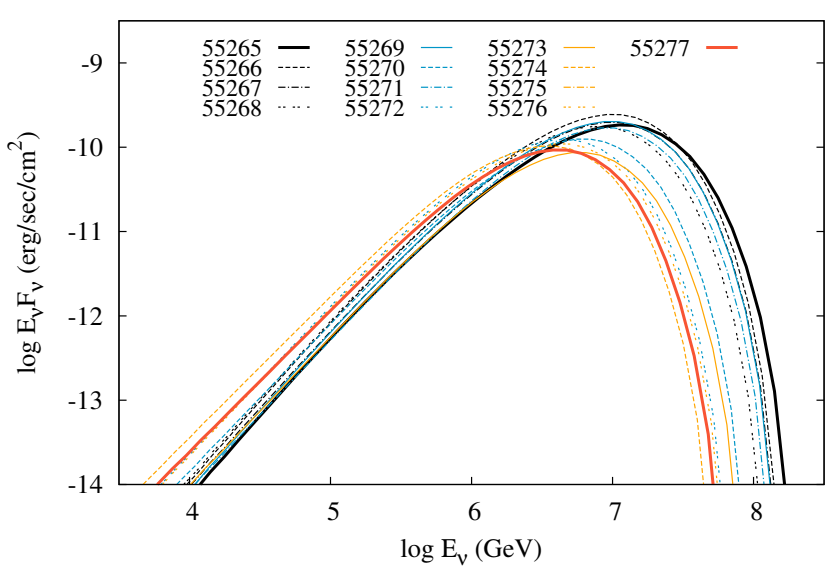

Figure 4: All-flavor neutrino $(v+\bar{v})$ fluxes derived by the model for the period MJD 55265-55277.

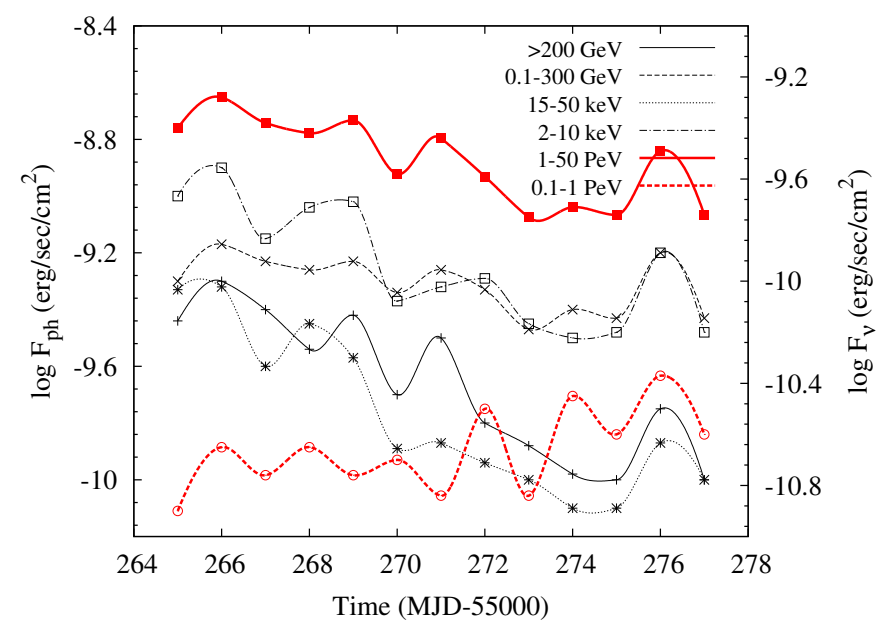

Figure 5: Model-derived light curves of Mrk 421 covering the period MJD 55265-55277. Symbols denote the daily fluxes photons $\left(F_{\mathrm{ph}}\right)$ and neutrinos $\left(F_{v}\right)$, while continuous lines are the result of interpolation. Photon light curves (black lines) are calculated at four energy bands (see inset legend). The allflavor neutrino $(v+\bar{v})$ light curves at 1-50 PeV and 0.1-1 PeV energy bands are also plotted with red solid and dashed lines, respectively. In all cases, the smooth curves are the result of interpolation.
Table 1: Pearson's correlation coefficient $r$ for the 1-50 PeV (0.1-1 PeV) neutrino flux $\left(F_{v}\right)$ vs. the photon flux $\left(F_{\mathrm{ph}}\right)$ in different energy bands. The null hypothesis is that the true correlation between the fluxes is non-zero.

\begin{tabular}{cccc}
\hline & Energy band & $r(11)^{\mathrm{a}}$ & Remark \\
\hline$F_{v}(1-50 \mathrm{PeV})$ & & & \\
\hline$F_{\mathrm{ph}}$ & $>200 \mathrm{GeV}$ & 0.97 & $\mathrm{~S}^{\mathrm{b}}$ \\
& $0.1-300 \mathrm{GeV}$ & 0.94 & $\mathrm{~S}$ \\
& $15-50 \mathrm{keV}$ & 0.89 & $\mathrm{~S}$ \\
& $2-10 \mathrm{keV}$ & 0.93 & $\mathrm{~S}$ \\
\hline$F_{v}(0.1-1 \mathrm{PeV})$ & & & \\
\hline$F_{\mathrm{ph}}$ & $>200 \mathrm{GeV}$ & -0.50 & $\mathrm{NS}$ \\
& $0.1-300 \mathrm{GeV}$ & -0.00 & $\mathrm{NS}$ \\
& $15-50 \mathrm{keV}$ & -0.43 & $\mathrm{NS}$ \\
& $2-10 \mathrm{keV}$ & -0.26 & $\mathrm{NS}$ \\
\hline
\end{tabular}

a The degrees of freedom (dof=N-2) for the Pearson's correlation significance test is given in the parenthesis.

b For $\mathrm{N}=13$, an observed value of $r$ larger than \pm 0.55 is statistically significant $(\mathrm{S})$ at a $5 \%$ level for a non-directional hypothesis; otherwise, the correlation is non-significant (NS).

The low-energy $(<1 \mathrm{PeV})$ part of the spectrum remains approximately constant in flux and spectral shape and is in good approximation independent of the $\gamma$-ray spectral variations, whereas the high-energy $(>1 \mathrm{PeV})$ neutrino spectrum is variable. Its relation to the photon flux is investigated below. Figure 5 shows the time evolution of the photon (black symbols/lines) and neutrino (red symbols/lines) fluxes in different energy bands as derived by modeling the daily SEDs of Mrk 421. In particular, the daily fluxes are shown as symbols, while continuous lines are the result of interpolation. As a posterior check of our SED modeling, we verified that the relation between the VHE $\gamma$-ray and X-ray (2-10 keV) fluxes is linear, in agreement with the results reported in [50]. The figure reveals signs of a correlation between the high-energy neutrino flux and the photon fluxes in all energy bands. To quantify these findings, we performed a Pearson's correlation test (see Table 1). The results show that the correlation between the 1-50 PeV neutrino flux and photon fluxes, in all energy bands, is statistically significant for a non-directional hypothesis at a 5\% level. The strongest correlation is found for the $1-50 \mathrm{PeV}$ neutrino flux and the VHE $(>200 \mathrm{GeV})$ photon flux, with a Pearson's correlation coefficient $r=0.97$. Furthermore, the high-energy neutrino flux (in logarithmic units) can be adequately described by a linear function of the logarithmic photon flux. For the $0.1-300 \mathrm{GeV}$ flux, in particular, we find

$$
\log F_{v}=A \log F_{\gamma}+B
$$

where $F_{\gamma}$ is defined as the $\gamma$-ray flux in the $0.1-300 \mathrm{GeV}$ energy band, $A=1.59 \pm 0.17$ and $B=5.25 \pm 1.64$. The relation between $F_{v}$ and $F_{\gamma}$ is of particular interest for the estimation of the cumulative neutrino event number within the five years of full IceCube livetime (see $§ 5$ ). 
Table 2: Expected IceCube neutrino event rate for the $v_{\mu}+\bar{v}_{\mu}$ daily SEDs shown in Fig. 4 compared to the background event count rate. For the point spread function, a $90 \%$ angular resolution of $1^{\circ}$ was assumed. All neutrino event rates are in units of yr ${ }^{-1}$ assuming a good IceCube runtime of 333 days per year, same as for the most recent point source data [53].

\begin{tabular}{ccccc}
\hline & \multicolumn{2}{c}{ Mrk 421 $^{\mathrm{a}}$} & \multicolumn{2}{c}{ Background $^{\mathrm{b}}$} \\
\hline$E_{v}(\mathrm{TeV})$ & $\begin{array}{c}\text { 13-day flare } \\
(55265-55277)\end{array}$ & $\begin{array}{c}\text { quiescent } \\
(54850-54983)\end{array}$ & atmospheric & diffuse \\
\hline $0.1-100$ & 0.023 & 0.019 & 7.371 & 0.010 \\
$100-10^{3}$ & 0.264 & 0.282 & $1.852 \times 10^{-3}$ & $2.203 \times 10^{-3}$ \\
$10^{3}-5 \times 10^{4}$ & 0.306 & 0.288 & $4.554 \times 10^{-6}$ & $2.236 \times 10^{-4}$ \\
\hline
\end{tabular}

a $90 \%$ of the signal flux is expected to be within $\Delta \Psi<1^{\circ}$.

${ }^{\mathrm{b}}$ Integrated over the bin-size $\Delta \Psi<1^{\circ}$.

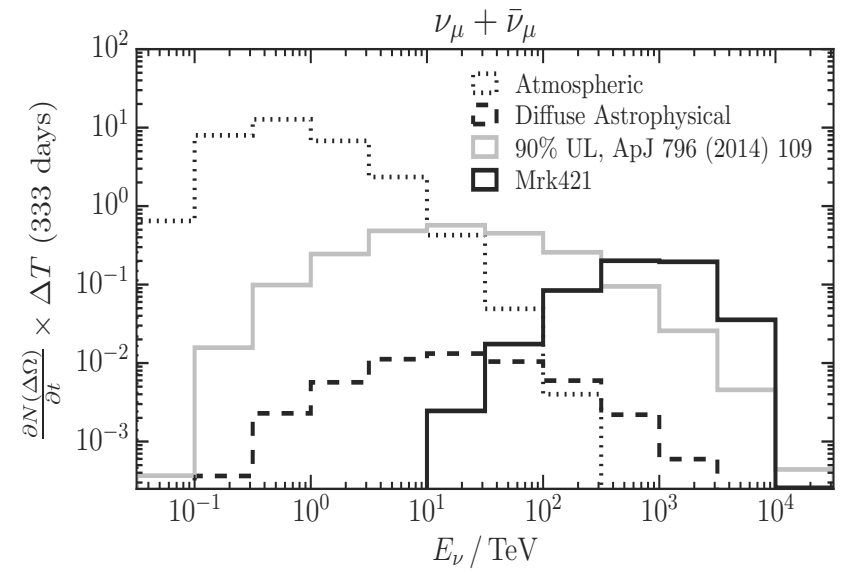

Figure 6: Differential (in energy) $v_{\mu}+\bar{v}_{\mu}$ event counts for 333 days of livetime calculated using eq. (1). Three different components are shown: the atmospheric neutrinos produced in decays of charged $\pi^{ \pm} / K^{ \pm}$(dotted line), the astrophysical component observed by IceCube (dashed line) [43, 52], and the model-derived $v_{\mu}+\bar{v}_{\mu}$ neutrino flux of Mrk 421 for the quiescent period MJD 54850-54983 (solid black line). The grey line shows the current IceCube upper limit calculated using an unbroken $E^{-2}$ power-law spectrum [53]. The observation window size corresponds to $\Delta \Omega=1^{\circ}$. Data are adopted from [53].
Using the model-derived daily neutrino fluxes ${ }^{3}$ shown in Fig. 4 and after taking into account the background neutrino fluxes, as described above, we calculate the expected event rates $\dot{N}_{v}$ using eq. (1) at different neutrino energy bins: 0.1-100 TeV, 0.1-1 PeV and 1-50 PeV. The results for the 13-day flare are summarized in Table 2. For comparison reasons, we also included the expected differential event rate $\dot{N}_{v}^{q}$ for the quiescent period MJD 54850-54983. The hard neutrino spectra predicted by the model for Mrk 421 suggest that most of the signal neutrinos should be observed at high energies, e.g. $\sim 0.57 \mathrm{evt} / \mathrm{yr}$ (events per year) above $100 \mathrm{TeV}$. At these energies, the neutrino background (atmospheric plus astrophysical) is negligible due to the soft energy spectrum and small observation window, thus making a potential neutrino signal from Mrk 421 a significant component.

One should note, though, that at energies above $1 \mathrm{PeV}$ Earth absorption starts to affect the incident neutrino flux. This is illustrated in Fig. 6 (see also Fig. 2), where the differential (in energy) $v_{\mu}+\bar{v}_{\mu}$ event counts for 333 days of livetime calculated using eq. (1) for the quiescent state of Mrk 421, are compared against those from various backgrounds. The $90 \%$ upper limit for Mrk 421 as obtained by IceCube [53] is also plotted (grey histogram) for comparison reasons. This is calculated assuming an unbroken $E^{-2}$ power-law neutrino spectrum. Being much steeper than our model-predicted spectra, it yields less events above $\sim 300 \mathrm{TeV}$ but predicts significantly more neutrinos in the $\mathrm{TeV}-300 \mathrm{TeV}$ region, where IceCube shows the best performance regarding point source searches (Fig. 3 in [53]).

A comparison between the 13-day flare and the quiescent period reveals a net gain in the expected neutrino event rate of the flare, at least for $E_{v}>1 \mathrm{PeV}$. This is, however, compensated by a relative loss at energies $0.1-1 \mathrm{PeV}$, thus leading to an approximately constant neutrino event rate at energies $E_{v}>100 \mathrm{TeV}$. Although the $\gamma$-ray activity of the source during the 13-day flare is high, e.g. the VHE $\gamma$-ray flux varies by a factor of $\sim 4$ (see Fig. 5) with a peak flux reaching $\sim 2$ Crab units [50], the neutrino event rate of the flare in the backgroundsuppressed regime is similar to the event rate of the longer and

\footnotetext{
${ }^{3}$ The neutrino flux produced at the source contains neutrinos of different flavors with an approximate ratio $F_{v_{\mathrm{e}}}: F_{v_{\mu}}: F_{v_{\tau}}=2: 1: 0$. However, by the time they reach Earth their ratio will have changed to $F_{v_{\mathrm{e}}}: F_{v_{\mu}}: F_{v_{\tau}}=1: 1: 1$ due to neutrino oscillations [74].
} 


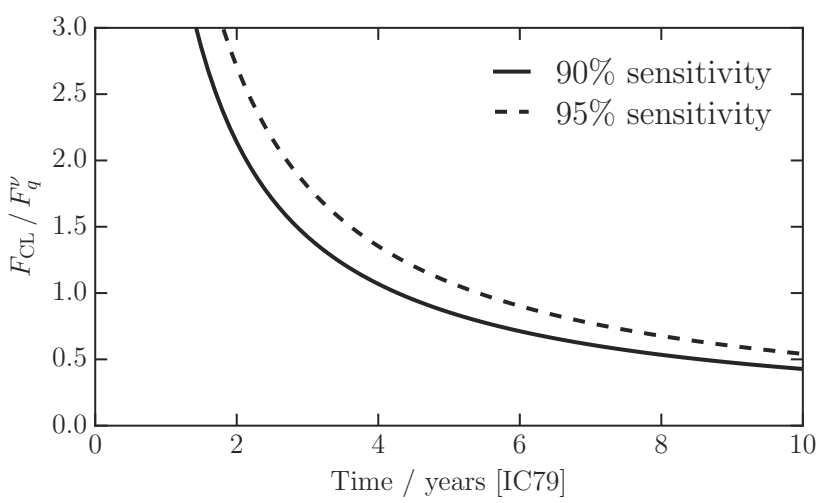

Figure 7: Muon neutrino flux $F_{v}$ from Mrk 421 (in units of the quiescent neutrino flux $F_{v}^{q}$ ) as a function of the time needed for IceCube to observe neutrinos with energy $E_{v}>100 \mathrm{TeV}$ at $90 \%$ (95\%) confidence level. Time is measured in years with respect to the start date of IC79 ( MJD 55348).

\section{The long-term $\gamma$-ray activity}

During the 13-day flaring period of 2010 the neutrino flux above $1 \mathrm{PeV}$ was found to correlate with the photon flux in all lation is present in longer time periods as well, we may apply

non-flaring period. We finally note that the biggest relative gain is observed at lower energies $\left(E_{v}<100 \mathrm{TeV}\right)$ where the high atmospheric background reduces, however, the sensitivity.

In the high-energy regime $\left(E_{v}>100 \mathrm{TeV}\right)$ a mean rate $\dot{N}_{v} \sim$ $0.57 \mathrm{evt} / \mathrm{yr}$ is expected over a negligible background rate of 0.04 evt/yr. Neglecting the small background, events originating from Mrk 421 will be detected at $90 \%$ confidence, as soon as the expected total number of events $N_{v}=\dot{N}_{v} \times T>2.3$, where is the neutrino event rate and $T$ is the observation time. Figure 7 shows the flux scaling needed for the models shown in Fig. 4 in order to observe at least one event from Mrk 421 at 90\% (solid line) and 95\% (dashed line) confidence level (CL). Given the IceCube event rate derived from the quiescent flux $F_{\gamma}^{q}$, we find that IceCube should observe events above $100 \mathrm{TeV}$ the 79-string IceCube (IC79) detector operation at 90\% (95\%) CL. the derived linear relation between the logarithmic neutrino and photon fluxes to the long-term light curve at a specific energy band, in order to calculate the expected number of muon neutrino events at $E_{v} \geq 1 \mathrm{PeV}$ within the five years of full IceCube detector livetime. ${ }^{4}$

Although the strongest correlation was derived for the VHE $\gamma$-ray and high-energy neutrino fluxes, here we choose to perform our analysis on the long-term Fermi-LAT (0.1-300 GeV)

\footnotetext{
${ }^{4}$ Here, the full IceCube livetime is defined with respect to the start of operation with 79 strings in May/June 2010 (IC79), i.e. before the completion of the detector one year later with 86 strings.
}

light curve of Mrk 421. The reason for doing so is that the Fermi-LAT light curve (MJD 54686.15-57192.15) covers the period of the complete IceCube operation, whereas the available long-term light curves in VHE $\gamma$-rays extend at most up to 2009 (for details, see [41]).

Figure 8 (top panel) shows the long-term (2506 d), weekly binned $\gamma$-ray light curve as observed with Fermi-LAT. The quiescent period of 2009 and the 13-day flare of 2010 are highlighted with grey and red symbols, respectively. Starting with a major $\gamma$-ray flare detected by Fermi-LAT in summer of 2012 [75], Mrk 421 entered a prolonged, still on-going, high-state period. During this period, at least four flares with $\gamma$-ray fluxes up to 3-10 times higher than that of the 2010 flare can be identified (light blue symbols). Henceforth, these will be referred to as 'major' flares. Inspection of the Fermi-LAT light curve alone would question the definition of the period MJD 55265-55277 as a flare. Although no significant variability was detected in the Fermi-LAT energy band (see also Fig. 5), the X-ray and VHE $\gamma$-ray variability was remarkable [50] (see also Figs. 3 and A.10).

Each of the major flares was fitted with a Lorentzian function and its characteristic duration was defined as twice that corresponding to its FWHM. The derived times of the peak fluxes and the respective flare durations are listed below:

- Flare 1a: MJD $56122.9 \pm 26.9$

- Flare 1b: MJD $56155.7 \pm 43.9$

- Flare 2: MJD 56393.09 \pm 34.04

- Flare 3: MJD $56767.2 \pm 47.6$

- Flare 4a: MJD 56942.3 \pm 62.3

- Flare 4b: MJD $56992.5 \pm 79.9$

We note that flares $1 \mathrm{a}$ and $1 \mathrm{~b}$ partially overlap and can be considered as one major double-peaked flare (see also [76]). The same applies to the latest flare. Its long duration as determined by a single Lorentzian fit to the data, suggests that this consists of, at least, two overlapping flares, here noted as Flares 4a and $4 \mathrm{~b}$.

The expected number of muon neutrino events can be then calculated as follows. The average $\gamma$-ray flux of the quiescent period MJD 54850-54983 is defined as

$$
F_{\gamma}^{q} \equiv \frac{1}{T_{q}} \int_{T_{q}} d t F_{\gamma}(t),
$$

where $T_{q}=133 \mathrm{~d}$ and $F_{\gamma}^{q}=4.199_{-0.165}^{+0.175} \times 10^{-10} \mathrm{erg} \mathrm{cm}^{-2} \mathrm{~s}^{-1}$ (for the error calculation, see Appendix B). The model-predicted $v_{\mu}+\bar{v}_{\mu}$ event rate above $1 \mathrm{PeV}$ for the quiescent period of Mrk 421 is $\dot{N}_{v}{ }^{q}=0.288$ evt/yr (see also Table 2). If $\dot{N}_{v}$ is the neutrino event rate for the period $T$, then $\dot{N}_{v}=\dot{N}_{v}^{q} F_{v} / F_{v}^{q}$, where $F_{v}^{q}, F_{v}$ are the average neutrino fluxes for the periods $T_{q}$ and $T$, respectively, and are defined similarly to eq. (3). According to the linear correlation derived in the previous section, the $\gamma$-ray and neutrino fluxes are related as $\log F_{v}=A \log F_{\gamma}+B$. Thus, 

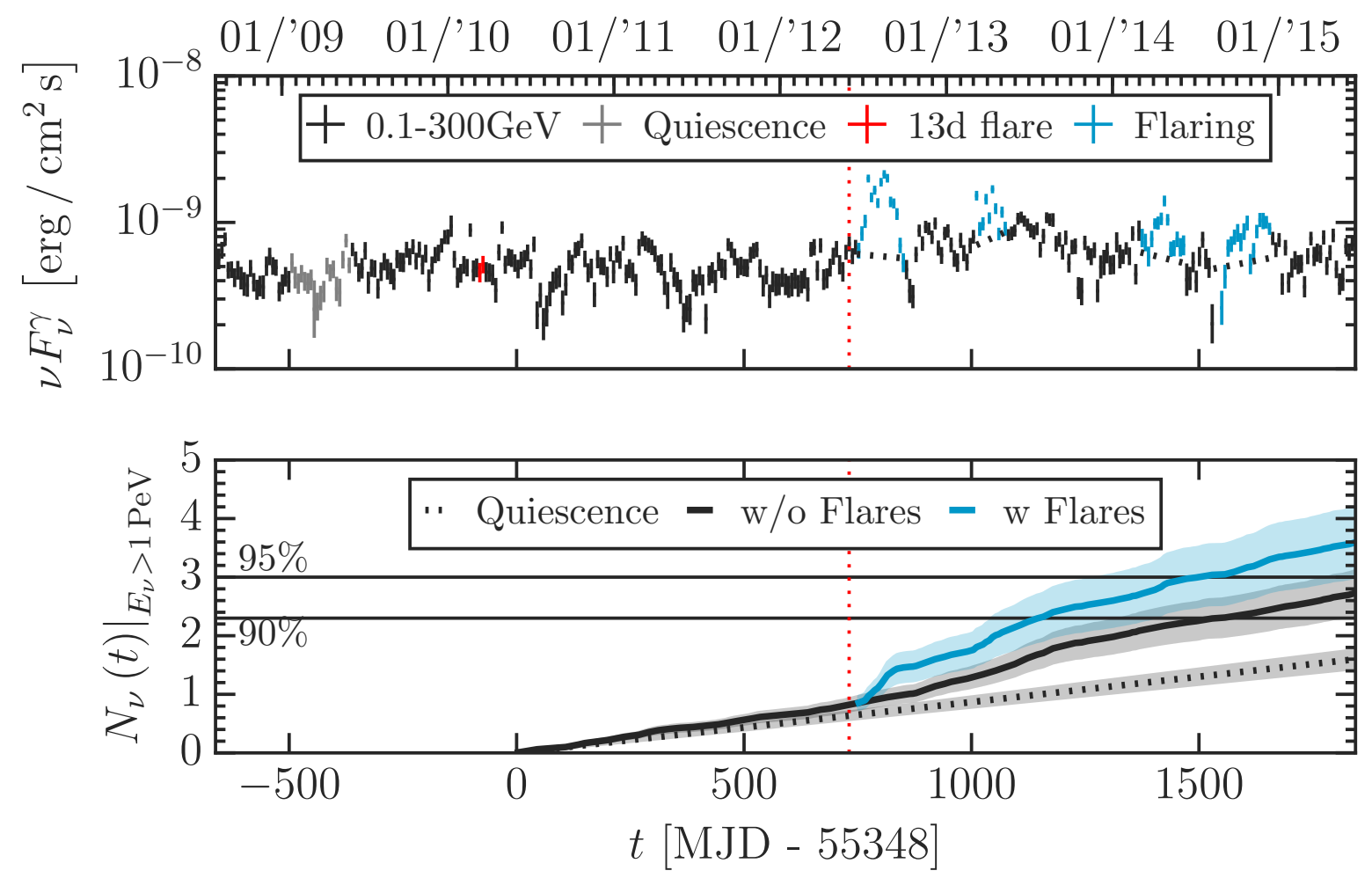

Figure 8: Top panel: Long-term, weekly binned $\gamma$-ray light curve of Mrk 421 at $0.1-300 \mathrm{GeV}$ as observed with Fermi-LAT. The quiescent period and the 13-day flare are highlighted with grey and red symbols, respectively. At least four major flares (for the definition, see text) can be identified (light blue symbols). Bottom panel: The cumulative number of muon neutrino events above $1 \mathrm{PeV}$ expected for IceCube within time $t$. The calculation is performed using the $v_{\mu}+\bar{v}_{\mu}$ flux estimated by the $\gamma$-ray light curve (top panel). The $v_{\mu}+\bar{v}_{\mu}$ flux is assumed to correlate with the $\gamma$-ray flux according to eq. (2). The cumulative curves obtained with and without the major flares included in the analysis are plotted with thick light blue ('w flares') and black ('w/o flares') lines, respectively. The results of the 'quiescent analysis', where a constant $\gamma$-ray flux, equal to that of the quiescent period (grey points in top panel) is assumed, are also plotted for comparison (dotted line). The latter is also the cumulative curve for neutrinos with energies $100 \mathrm{TeV}$ - 1 PeV. In all cases, the 90\% uncertainties on the mean expected count are shown as shaded bands. These take into account the systematic uncertainties of the IceCube effective area, the statistical uncertainties of the $\gamma$-ray observations, and the error of the slope in the relation $\log F_{v}-\log F_{\gamma}$. Horizontal lines indicate the threshold for the observation of one or more neutrinos at $90 \%$ or $95 \%$ CL. The latest published results of IceCube [53] included data until MJD 56063, which is marked by the vertical red-dotted line. 
using the long-term Fermi-LAT light curve, we may estimate the number of events expected in time period $T$ as

$$
N_{v} \equiv \dot{N}_{v} T=\frac{\dot{N}_{v}^{q}}{F_{v}^{q}} \int_{T} d t F_{v}(t)=\dot{N}_{v}^{q} \int_{T} d t\left(\frac{F_{\gamma}(t)}{F_{\gamma}^{q}}\right)^{A} .
$$

As the $\gamma$-ray light curve of Mrk 421 is weekly binned, the integral of eq. (4) can be approximated by a sum with bin-width $\Delta t=7 \mathrm{~d}$ :

$$
N_{v}=\frac{\dot{N}_{v}^{q}}{F_{v}^{q}} \int_{T} d t F_{v}(t)=\frac{\dot{N}_{v}^{q} \Delta t}{\left(F_{\gamma}^{q}\right)^{A}} \sum_{i}\left(F_{\gamma, i}\right)^{A} .
$$

The logarithmic event count $n_{v}=\log N_{v}$ then yields

$$
n_{v}=\log \left(\Delta t \dot{N}_{v}^{q}\right)+\log \sum_{i}\left(F_{\gamma, i}\right)^{A}-A \log F_{\gamma}^{q},
$$

and the respective error is given by

$$
\sigma_{n_{v}}^{2}=f_{\dot{N}_{v}^{q}}^{2}+f_{F_{\gamma, i}}^{2}+f_{F_{\gamma}^{q}}^{2}+f_{\mathrm{A}}^{2}
$$

where the various contributions to the total uncertainty are

$$
\begin{aligned}
f_{\dot{N}_{v}^{q}} & =\frac{\sigma_{\dot{N}_{v}^{q}}}{\ln 10 \dot{N}_{v}^{q}} \\
f_{F_{\gamma, i}} & =\frac{\sqrt{\sum_{i}\left(\sigma_{F_{\gamma, i}} A F_{\gamma, i}^{A-1}\right)^{2}}}{\ln 10 \sum_{i} F_{\gamma, i}^{A}} \\
f_{F_{\gamma}^{q}} & =\frac{A \sigma_{F_{\gamma}^{q}}}{\ln 10 F_{\gamma}^{q}} \\
f_{\mathrm{A}} & =\frac{\sigma_{A} \sum_{i} F_{\gamma, i}^{A} \ln \frac{F_{\gamma, i}}{F_{\gamma}^{q}}}{\ln 10 \sum_{i} F_{\gamma, i}^{A}} .
\end{aligned}
$$

In the above, $\sigma_{\dot{N}_{v}^{q}}$ is the uncertainty of the muon neutrino event rate in quiescence, which is dominated by systematic effects of the IceCube detector, and is accounted for with $10 \%$ relative uncertainty [53]. Furthermore, $\sigma_{F_{\gamma, i}}$ is the statistical error of the $\gamma$-ray flux measurements and $\sigma_{F_{\gamma}^{q}}$ is the uncertainty of the $\gamma$-ray flux in quiescence (for the derivation, see Appendix B). Finally, $\sigma_{A}$ is the $1 \sigma$ error of $A$ as obtained from fitting the modeled neutrino and 0.1-300 GeV $\gamma$-ray fluxes (see eq. (2)).

Using eqs. (5), (6) and (7), we calculated $N_{v}$ in three different cases described below. First, we performed the analysis using the full Fermi-LAT light curve ('w flares' analysis). Then, to exemplify the net effect of the major flares on the expected number of muon neutrino events, we performed the analysis after excluding the major flares ('w/o flares' analysis). However, in order to include the respective exposure time in this analysis, we interpolated the $\gamma$-ray light curve using mean fluxes in the time just before and after the end of each major $\gamma$-ray flare (see black dashed lines in top panel of Fig. 8). Finally, we considered the extreme case of a non-variable $\gamma$-ray light curve with flux equal to that of the 2009 quiescent period ('quiescent' analysis).

The cumulative event count $N_{v}$ expected for IceCube from Mrk 421 is presented in the bottom panel of Fig. 8, where the results of the different analyses are plotted with different types

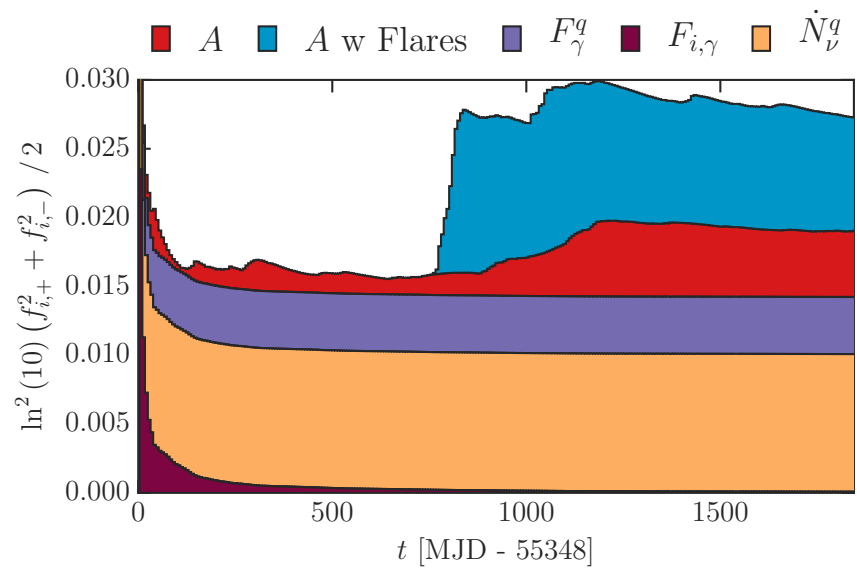

Figure 9: Stacked contributions of the various sources of uncertainty $f_{i}^{2}$ defined in eqs. (8)-(11) that add up to the total uncertainty given by eq. (7). In addition, the uncertainty on the slope $A$ of the $\log F_{v}-\log F_{\gamma}$ relation is shown for the two scenarios, i.e. with and without major flares in blue and red, respectively. For all other sources of uncertainty, the differences between the two scenarios are negligible. of lines (for details, see figure caption). The total uncertainty given by eq. (7) is shown, in each case, as a shaded band around the mean event count. The various sources of uncertainty that contribute to the total one are presented in Fig. 9. The major contributions to $\sigma_{n_{v}}^{2}$ originate from the systematic uncertainty of $A_{\text {eff }}$ (yellow pale color) and from the uncertainty on the $\log F_{v}-\log F_{\gamma}$ relation. Although the latter is sub-dominant in the analysis 'w/o flares' (red color) compared to the systematic uncertainty, it becomes comparable to it when the major flares are included (blue color). The statistical uncertainty of the $\gamma$-ray flux measurements is similar in both analyses, while it becomes negligible as the total observing time in $\gamma$-rays increases.

From Fig. 8 (bottom panel), it becomes evident that the inclusion of major $\gamma$-ray flares greatly increases the event number, as long as the derived correlation of the $\gamma$-ray flux with the $>1 \mathrm{PeV}$ neutrino flux still holds. To better quantify these results, we present in Tables 3 and 4 the expected $v_{\mu}+\bar{v}_{\mu}$ event numbers for each one of the IceCube operation seasons and major flares, respectively ${ }^{5}$. We find that the event rate during Flares $1 \mathrm{a}$ and $1 \mathrm{~b}$ exceeds the event rate for the same year $(06 / 2012-05 / 2013)$ by a factor $3.0 \pm 0.9$. Although the probability to observe at least one muon neutrino event during Flares 1a and 1b is $46 \%$ (see Table 4), a restricted neutrino search over the period of the 2012 major flare would not guarantee a neutrino detection. In contrast, $1.46 \pm 0.32$ $v_{\mu}+\bar{v}_{\mu}$ events are expected within the period covered by all four major flares (385 days in total). In this regard, stacked analyses of major flares from Mrk 421 would be more beneficial for neutrino searches.

\footnotetext{
${ }^{5}$ The respective numbers of $v_{\mu}+\bar{v}_{\mu}$ events with $100 \mathrm{TeV}<E_{v}<1 \mathrm{PeV}$ can be easily obtained from the values listed in Table 2 and the duration of each operation season.
} 
Table 3: Number of high-energy $v_{\mu}+\bar{v}_{\mu}$ events $\left(E_{v}>1 \mathrm{PeV}\right)$ expected for IceCube in various seasons of operation (each with duration $\mathrm{T}$ in days). The results are obtained using the FermiLAT $\gamma$-ray light curve in Fig. 8 (top panel) and the connection to the high-energy neutrino flux through eq. (4). The values for each season are obtained after replacing the major $\gamma$-ray flares (Table 4) with a non-variable emission, whose flux was determined through interpolation of the $\gamma$-ray light curve just before the start and after the end of each major flare (for details, see text). The total number of events without (with) the major flares included are also presented. For each entry, the probability $P_{N_{v} \geq 1}$ of observing one or more neutrinos is quoted.

\begin{tabular}{cccc}
\hline Season & T (days) & $v_{\mu}+\bar{v}_{\mu}$ & $P_{N_{v} \geq 1}(\%)^{\dagger}$ \\
\hline $06 / 2010-05 / 2011$ & 364 & $0.43 \pm 0.06$ & $34 \pm 4$ \\
$06 / 2011-05 / 2012$ & 364 & $0.38 \pm 0.05$ & $32 \pm 3$ \\
$06 / 2012-05 / 2013$ & 371 & $0.71 \pm 0.11$ & $51 \pm 5$ \\
$06 / 2013-05 / 2014$ & 364 & $0.70 \pm 0.11$ & $50 \pm 5$ \\
$06 / 2014-05 / 2015$ & 350 & $0.47 \pm 0.06$ & $38 \pm 4$ \\
\hline$\sum$ w/o Flares & $1834^{\mathrm{a}}$ & $2.73 \pm 0.38$ & $94 \pm 2$ \\
$\sum$ w Flares & 1834 & $3.59 \pm 0.60$ & $97 \pm 2$ \\
\hline
\end{tabular}

$\dagger$ Using Poisson statistics, $P\left(N_{v} \geq 1\right)=1-\mathrm{e}^{-\lambda}$ for a Poisson distribution with mean $\lambda$.

a On top of the quoted years, three weeks of additional

Fermi-LAT data are available after 05/2015.

These results, albeit model-dependent, demonstrate that major (in duration and flux) flares from blazars like Mrk 421 could serve as favorable periods for time-dependent neutrino searches. until MJD 56063 (red dotted line in Fig. 8), that is just before the major flare of Mrk 421 in 2012.

Excluding the flares as explained previously, the expected number of $v_{\mu}+\bar{v}_{\mu}$ events up to the date where the most recent IceCube data are available (05/2015), is found to be $2.73 \pm 0.38$ tection within the uncertainties. The respective number in the which excludes a non-detection of neutrinos by more than $95 \%$.

By utilizing the neutrino-photon flux correlation, we found a significant increase in the expected neutrino rate compared to that obtained from the quiescent state alone. In particular, the prediction of the quiescent state would yield $\sim 1.6$ events in the IceCube livetime, thus underestimating the neutrino event rate after June 2012. Since then, Mrk 421 entered a high $\gamma$-ray flux period that is still on-going (see top panel in Fig. 8). We remark that the estimate of 1.6 events applies also to neutrinos with energies in the range $100 \mathrm{TeV}-1 \mathrm{PeV}$, since we found no correlation between the photon and sub-PeV neutrino fluxes in our modeling of the 13 day flare (see Table 1). In this regard, the estimate derived from the quiescent state is the most robust.

\section{Discussion}

By modeling the daily SEDs of Mrk 421 we were able to derive the daily $v_{\mu}+\bar{v}_{\mu}$ neutrino fluxes and compare them against

Table 4: Same as Table 3 but for the four flares that were identified in this analysis.

\begin{tabular}{cccc}
\hline No. & T (days) & $v_{\mu}+\bar{v}_{\mu}$ & $P_{N_{v} \geq 1}(\%)$ \\
\hline Flares 1a+1b & 105 & $0.61 \pm 0.16$ & $46 \pm 8$ \\
Flare 2 & 70 & $0.32 \pm 0.07$ & $27 \pm 5$ \\
Flare 3 & 98 & $0.26 \pm 0.05$ & $23 \pm 4$ \\
Flares 4a+4b & 112 & $0.26 \pm 0.05$ & $23 \pm 4$ \\
\hline$\sum$ Flares & 385 & $1.46 \pm 0.32$ & $77 \pm 7$ \\
\hline
\end{tabular}

those obtained for the longer, albeit quiescent, period of 2009. Although one could naively argue that the neutrino event rate would be higher during the 13-day flare, we showed explicitly that the mean event rate above $100 \mathrm{TeV}$ is $\sim 0.57 \mathrm{evt} / \mathbf{y r}$ ( $\sim 0.26 \mathrm{evt} / \mathrm{yr}$ for $100 \mathrm{TeV}<E_{v}<1 \mathrm{PeV}$ and $\sim 0.31 \mathrm{evt} / \mathrm{yr}$ for $\left.E_{v}>1 \mathrm{PeV}\right)$. This is comparable to that expected for a fourmonth period of lower $\gamma$-ray and X-ray fluxes. Due to the short duration of the flare, the expected number of muon neutrino events is $\sim 0.02$, i.e. insufficient to explain a fiducial neutrino detection from the direction of Mrk 421 in the time-window of a flare with similar characteristics as the one studied here. Interestingly, [37] reached similar conclusions within a different leptohadronic model for explaining the orphan VHE $\gamma$-ray flare of blazar 1ES 1959+650.

Inspection of the Fermi-LAT light curve (top panel in Fig. 8) clearly shows a transition of the source to a period of increased $\gamma$-ray flux (i.e., high state) that was initiated in 2012 by a major flare, and is still on-going. Within this period we identified four major flares, in total, with peak fluxes $\sim 3-10$ times higher than the one modeled here. Whether the correlation between the neutrino and $\gamma$-ray fluxes we derived in $\$ 4.2$ still holds during these extreme flares cannot be safely answered without detailed modeling of the respective SEDs. As the SED is composed of many emission components (see Fig. 3), which are moreover dependent on each other, it is not trivial to predict the neutrino- $\gamma$-ray flux correlation during flares without having knowledge of the variability at lower energy bands; this was the motivation of this study in the first place. Consider for example a scenario where the major $\gamma$-ray flare is not accompanied by a respective increase of the X-ray flux. An increase of the proton injection luminosity, which would also lead to a higher neutrino flux, could not explain this fiducial flare, since the Bethe-Heitler component would also be enhanced, therefore violating the fiducial observations. More than one model parameter should be changed and, depending on their combination, the derived neutrino flux would also differ. Because of the wide range of possibilities, in the present study we simply assume that the relation given by eq. (2) is valid over the $6.9 \mathrm{yr}$ period of Fermi-LAT observations, while the major flare MW modeling and its implications for the neutrino flux will be the subject of a subsequent paper.

Nevertheless, in order to assess the net effect of the major flares on the predicted $v_{\mu}+\bar{v}_{\mu}$ event number we performed an additional analysis ('w/o flares'), where these were not taken into account. More precisely, in order to include the respective exposure times in our analysis, we replaced the high $\gamma$-ray 
fluxes of the major flares with values obtained from the inter-

polation of the $\gamma$-ray light curve just before the start and after the end of each major flare (see dashed lines in the top panel of Fig. 8). We showed that $1.46 \pm 0.32 v_{\mu}+\bar{v}_{\mu}$ events are expected within a period of 385 days due to the major flares alone. Thus, their presence increases the neutrino event rate within the IceCube livetime by $30 \%$ (see Tables 3 and 4). Furthermore, the neutrino rate without (with) the major flares, as estimated by the long-term $\gamma$-ray light curve, is $73 \%(127 \%)$ higher than that expected by simply extrapolating the neutrino flux in the quiescent state (see Table 1). In brief, the predictions for the cumulative event count above $1 \mathrm{PeV}$ are significantly affected by the major flares under the assumption of a neutrino- $\gamma$-ray flux relation given by eq. (2). The values derived from the 'quiescent' analysis ( $1.60 \pm 0.16$ events in 1834 days) apply, however, directly to the $100 \mathrm{TeV}-1 \mathrm{PeV}$ neutrino event counts, since we found no significant correlation between the sub-PeV and photon fluxes (in any energy band). Meanwhile, they constitute a robust lower bound for the predicted cumulative events above $1 \mathrm{PeV}$.

Based on four years of data searches using through-going muons, IceCube reported an overfluctuation of events at the position of Mrk 421 [53]. The best-fit to the data yielded $n_{\mathrm{s}}=3.8$ signal events over the full energy range for an unbroken powerlaw spectrum $d N_{v} / d E_{v} \propto E_{v}^{-\gamma}$ with $\gamma \sim 1.9$, while 22.4 background events were expected in a circle $1^{\circ}$ around the search coordinates. This result deviates from the atmospheric background expectation of a soft spectrum with $\gamma \sim 3.7$, but is still consistent with a pure background expectation $(p \approx 26 \%$, where $p$ is the pre-trial probability). The $90 \%$ upper limit on the flux normalization of an unbroken $E^{-2}$ flux was set to $\Phi_{v_{\mu}+\bar{v}_{\mu}}^{90 \%}=$ $2.1 \times 10^{-12} \mathrm{TeV}^{-1} \mathrm{~cm}^{-2} \mathrm{~s}^{-1}$. As Fig. 6 demonstrates, such a soft spectrum yields events mostly at the $\mathrm{TeV}$ energy range, whereas the model adopted in this study predicts a spectrum peaking at the PeV energy range. Two out of the four years used in [53, 77] are in full detector configuration, thus coinciding with our calculations for the long-term light curve of Mrk 421 (see §5). Within the overlapping period of 728 days, $0.81 \pm 0.08 v_{\mu}+\bar{v}_{\mu}$ events are expected above PeV energies and $0.62 \pm 0.06$ for energies between $100 \mathrm{TeV}$ and $1 \mathrm{PeV}$ (see Table 3). These results do not contradict the observation of IceCube that the overfluctuation is consistent with pure background. A fit to more recent IceCube data that include two additional years [78] increased the number of signal events to $n_{\mathrm{s}}=5.5$. Yet, this is still consistent with the background expectation.

Within the period of five years, and according to our estimations, IceCube is expected to detect high-energy $\left(E_{v}>1 \mathrm{PeV}\right)$ neutrinos from Mrk 421 at $90 \%$ confidence level. Hence, with additional data, IceCube's sensitivity will surpass our model predictions, thus testing scenarios of cosmic-ray acceleration at the PeV energy regime. Even a non-detection of neutrinos would be of great importance, though; this can place constraints on the contribution of the hadronic component to the high-energy emission from Mrk 421, as we illustrate below. Given a non-detection in $X$ years, the most robust constraint on the hadronic contribution of Mrk 421 can be derived
Table 5: Upper limits on the proton luminosity in the blob as derived from a non-detection (at $90 \%$ and $95 \% \mathrm{CL}$ ) of muon neutrinos (> $100 \mathrm{TeV}$ ) from Mrk 421 in $X$ years.

\begin{tabular}{ccccc}
\hline$X(\mathrm{yr})$ & \multicolumn{2}{c}{$\zeta_{X}$} & \multicolumn{2}{c}{$L_{\mathrm{p}, \mathrm{X}}(\mathrm{erg} / \mathrm{s})$} \\
& $90 \%$ & $95 \%$ & $90 \%$ & $95 \%$ \\
\hline 6 & 0.71 & 0.9 & $6.2 \times 10^{47}$ & $7.8 \times 10^{47}$ \\
8 & 0.53 & 0.68 & $4.6 \times 10^{47}$ & $5.9 \times 10^{47}$ \\
10 & 0.43 & 0.54 & $3.7 \times 10^{47}$ & $4.7 \times 10^{47}$ \\
20 & 0.21 & 0.27 & $1.8 \times 10^{47}$ & $2.3 \times 10^{47}$ \\
\hline
\end{tabular}

from the quiescent scenario (see e.g. Fig. 7). The rest of our predictions (see Tables 3 and 4 ) are based on the assumption of a correlation $F_{v} \propto F_{\gamma}^{A}$, whose long-term validity may be questionable. In order to be able to constrain the hadronic component in flaring periods, one should first test the validity of the correlation in different epochs of flaring activity through SED modeling. A smaller number of neutrinos implied by a non-detection in $X$ years could be obtained either by an absent correlation or a lower proton luminosity in the blob. Hence, in the following, we focus on the quiescent scenario where the constant $v_{\mu}+\bar{v}_{\mu}$ flux is related to the proton luminosity as $F_{v}=(1 / 4) f L_{\mathrm{p}} / 4 \pi d_{\mathrm{L}}^{2}$, where we assumed that $50 \%$ of the interactions lead to $\pi^{0}$ production and $L_{\mathrm{p}}$ is the proton luminosity in the blob as measured in the observer's frame. Moreover, $f$ is the pion production efficiency which may be written as (see e.g. [65])

$$
f \approx 2.2 \times 10^{-3} L_{\mathrm{s}, 45} R_{15}^{-1} v_{\mathrm{s}, 16}^{-1} \delta_{1}^{-3}
$$

where $L_{\mathrm{s}}$ is the apparent bolometric luminosity of the lowenergy hump of the SED and $v_{\mathrm{S}}$ is the respective peak frequency. Here, the notation $q_{\mathrm{X}} \equiv q / 10^{\mathrm{X}}$ has been introduced. For parameters relevant to the quiescent period $f \approx 10^{-5}$, in agreement with previous studies on BL Lac neutrino emission (e.g. [25, 33, 79]). A non-detection of muon neutrinos above $100 \mathrm{TeV}$ in $X$ years translates into $F_{v, \mathrm{X}}=\zeta_{\mathrm{X}} F_{v}^{(\mathrm{q})}$, where $F_{v}^{(\mathrm{q})} \simeq 2.4 \times 10^{-10} \mathrm{erg} \mathrm{cm}^{-2} \mathbf{s}^{-1}$ and $\zeta_{X} \leq 1$ can be read from the sensitivity curves shown in Fig. 7. Combination of the above leads to

$$
L_{\mathrm{p}, \mathrm{X}}=1740 \zeta_{\mathrm{X}} F_{\nu, \mu}^{(\mathrm{q})} 4 \pi d_{\mathrm{L}}^{2} L_{\mathrm{s}, 45}^{-1} R_{15} v_{\mathrm{s}, 16} \delta_{1}^{3} \mathrm{erg} \mathrm{s}^{-1} .
$$

Our results are summarized in Table 5 for two CL values.

All the estimates we have presented so far are based on the up-going muon sample, as this is the most relevant for point source searches, especially, those located in the northern sky (for details, see §3). The HESE sample, on the other hand, consists of a small, yet high purity, statistical sample of astrophysical all-flavors neutrinos due to the veto imposed on atmospheric events. Its high purity comes, though, at the cost of a largely reduced effective area (see e.g. Fig. 1). Thus, a meaningful comparison between the expected number of muon neutrinos listed in Tables 1-3 and depicted in Fig. 8 with those obtained from the five-year analysis of the HESE sample [80] would require an appropriate scaling of the rates by a factor of $\sim 50$, which is due to the difference in effective areas as shown in Fig. 1. For the most optimistic 
scenario considered here (' $w$ flares'), the expected number of $v_{\mu}+\bar{v}_{\mu}$ events in 5 years with the HESE analysis would read $\sim 3.59 / 50 \simeq 0.07$ or $\sim 0.2$ all-flavor events. These values are still consistent with the results reported in [80].

It is also worth noting that our hypothesis of the PeV neutrino$\gamma$-ray correlation during major flares can be further tested with specific, time-optimized analyses similar to those presented in [77]. Nevertheless, multiple flares or long-lasting flaring periods are needed to accumulate enough exposure for a neutrino detection. These can make use of the most recent IceCube data, since enhanced neutrino event rates are expected, based on our hypothesis, in the recent years of full IceCube detector operation. Furthermore, the long-term Fermi-LAT light curve of Mrk 421 implies that the quiescent state of 2009 may not be the most characteristic state of activity, since the blazar entered a long-lasting period of increased $\gamma$-ray flux since the summer of 2012. Consequently, the observation of Mrk 421 in search of high-energy neutrinos might be more efficient, when focused on periods where higher neutrino emission is expected.

There are two additional mechanisms of neutrino production implied by our model, which were not shown in detail because their contribution to the total spectrum is minimal. The first is neutron decay. High-energy neutrons, a by-product of photomeson interactions (e.g., $p+\gamma \rightarrow \pi^{+}+n$ ), are free to escape the emission region, thus providing an effective means of cosmic-ray escape [81-83], while producing at the same time $\bar{v}_{\mathrm{e}}$. As shown in DPM14, those neutrinos are lower in both energy and flux than the ones resulting from meson decays, by about two orders of magnitude. The second mechanism results from the $\beta$-decay produced protons propagating in the interstellar and intergalactic medium as cosmic rays, and interacting with ambient radiation fields [56], i.e. the extragalactic background light (EBL). In the present discussion we neglect any contribution to the cosmic-ray flux from direct proton escape [e.g. 84]. The highest-energy escaping protons considered in our model have energy $E_{\mathrm{p} \text {,max }}^{\text {esc }} \sim 1.6 \times 10^{8} \mathrm{GeV} \Gamma_{1.3}$, where $\Gamma \sim \delta$. These protons are energetic enough to pion-produce on photons with energy $E_{0} \gtrsim 0.9 \mathrm{eV} \Gamma_{1.3}^{-1}$. The present $(z=0)$ EBL energy density at $\sim 1 \mathrm{eV}$ is $u_{0} \simeq 4 \times 10^{-15} \mathrm{erg} \mathrm{cm}^{-3}$ [e.g. 85-87]. The photopion energy loss rate for protons with $E_{\mathrm{p}, \text { max }}^{\text {esc }}$ can be then estimated as $t_{\mathrm{p} \pi, \mathrm{EBL}}^{-1} \approx \kappa_{\mathrm{p} \pi} \sigma_{\mathrm{p} \pi} c n_{0} E_{0}$, where $n_{0}=u_{0} / E_{0}^{2}$, and $\kappa_{\mathrm{p}}=0.2$ and $\sigma_{\mathrm{p} \pi} \simeq 5 \times 10^{-28} \mathrm{~cm}^{2}$ are the inelasticity and cross section, respectively, at the $\Delta(1232)$ resonance [88]. The pion production efficiency on the EBL photons can be then estimated as $f_{\mathrm{EBL}} \equiv t_{\mathrm{cr}} / t_{\mathrm{p} \pi \text {,EBL }} \approx 10^{-4}$, where we conservatively used $t_{\mathrm{cr}}=d_{\mathrm{L}} / c$, assuming rectilinear proton propagation. The propagation of protons at these energies may be diffusive [e.g. 89], thus increasing the residence time of protons in the ISM, while isotropizing both the cosmic-ray and accompanying neutrino fluxes. Regardless, the net effect would be a decrease of the observed neutrino flux. The efficiency $f$ of pion production in the emission region of the blazar jet can be calculated in a similar way. By approximating the low-energy hump of the SED as a monochromatic photon field with characteristic frequency $v_{\mathrm{S}}$ and luminosity $L_{\mathrm{S}}$, it can be shown that $f \simeq 22 L_{\mathrm{s}, \mathbf{4 6}} / R_{15} v_{\mathrm{s}, 16} \delta^{3}$ Substitution of parameter values rele- vant to the modeling of the 13-day flare, namely $L_{\mathrm{S}, 46}=1$, $v_{\mathrm{s}, 16}=8, R_{15}=3.2$ and $\delta=20$, results in $f \approx 10^{-4}$. Interestingly, this is comparable to $f_{\mathrm{EBL}}$. Yet, the neutrino luminosity produced via photomeson interactions during the propagation in the ISM, $L_{v}^{\text {prop }}$, is expected to be much lower than that produced internally in the blazar emission region, $L_{v}^{\text {int }}$. The respective ratio is given by $L_{v}^{\text {prop }} / L_{v}^{\text {int }} \approx f_{\mathrm{EBL}} L_{\mathrm{p}}^{\text {esc }} / L_{v}^{\text {int }}$, where $L_{\mathrm{p}}^{\text {esc }} \equiv L_{\mathrm{n}}$. The neutron luminosity is, in turn, given by $L_{\mathrm{n}} \simeq$ $(20 / 6) L_{v}^{\mathrm{int}}$, where we assumed the production of $\pi^{ \pm}$in a single photopion interaction, leading to $n: v=1: 6$ and that $E_{\mathrm{n}} \simeq 20 E_{v}$ (see also, [90]). Combining all the above we find $L_{v}^{\text {prop }} / L_{v}^{\text {int }} \approx(20 / 6) f_{\mathrm{EBL}} \approx \mathbf{3} \times 10^{-4}<<1$.

\section{Summary}

We presented calculations of the expected neutrino emission from flaring periods of the nearby blazar Mrk 421 in the context of a one-zone leptohadronic model for its MW photon emission. In this scenario, protons are accelerated and subsequently injected in the emission region of the blazar, where they pion-produce on the internal synchrotron radiation emitted by a co-accelerated relativistic electron population. High-energy neutrinos, which are produced through the decay of charged mesons, are the final product of photopion interactions, and may escape the blazar unimpeded, thus providing the smoking gun for hadron acceleration in blazars.

Using a time-dependent, energy-conserving leptohadronic numerical code [48] we modeled the photon SEDs of the 13-day flare of 2010, which was the target of an unprecedented MW campaign [50]; the flaring episode was simultaneously (within 2 or 3 hours) observed from radio wavelengths up to the VHE $\gamma$-ray regime. Based on the model-derived daily neutrino spectra, we calculated the IceCube muon neutrino event rate of the 13-day flare, and showed that at energies $>100 \mathrm{TeV}$ it is comparable to the one expected from a longer but non-flaring period of Mrk 421, i.e. during quiescence. We concluded that an accumulation of similar flares over several years would be necessary to produce a meaningful signal for IceCube.

The detailed modeling of the 13-day flare revealed a strong correlation of the expected high-energy neutrino flux with the photon flux in various energy bands, ranging from soft X-rays $(\sim 2-10 \mathrm{keV})$ up to VHE $(>200 \mathrm{GeV}) \gamma$-rays. In particular, the relation between the high-energy neutrino and $0.1-300 \mathrm{GeV}$ photon fluxes was found to be $F_{v} \propto F_{\gamma}^{A}$ where $A \sim 1$.6. We applied the relation, assuming it is valid for longer time periods as well, to the long-term Fermi-LAT $\gamma$-ray light curve of Mrk 421 that spans $\sim 6.9$ years and coincides with the operation period of the full IceCube detector. We then estimated the cumulative number of $v_{\mu}+\bar{v}_{\mu}$ events above $1 \mathrm{PeV}$ for the full IceCube detector livetime, and found $3.59 \pm 0.60(2.73 \pm 0.38)$ events with (without) major flares included in our analysis. This estimate exceeds, within the uncertainties, the 95\% (90\%) threshold value for the detection of at least one neutrino event. Meanwhile, the most conservative scenario, where no correlation of $\gamma$-rays and neutrinos is assumed, predicted $1.60 \pm 0.16 v_{\mu}+\overline{v_{\mu}}$ events, still below the $90 \%$ IceCube sensitivity. 


\section{Acknowledgments}

In conclusion, by utilizing model predictions about the correlation of $\mathrm{PeV}$-neutrinos and $\gamma$-rays, experiments like IceCube can focus their neutrino searches on well-monitored sources, such as Mrk 421, and stack periods where high neutrino activity is expected, such as in major $\gamma$-ray flares.

We thank Prof. E. Resconi and Prof. A. Mastichiadis for useful comments on the manuscript. We thank Dr. T. Hovatta for providing us with the Fermi-LAT light curve. We also thank the anonymous referees for their comments and suggestions. M.P. acknowledges support for this work by NASA through Einstein Postdoctoral Fellowship grant number PF3 140113 awarded by the Chandra X-ray Center, which is operated by the Smithsonian Astrophysical Observatory for NASA under contract NAS8-03060. S.C. acknowledges support by the cluster of excellence "Origin and Structure of the Universe".

\section{Appendix A. Daily SEDs for the period MJD 55266-55276 and model parameter values}

In the figures that follow we present the model fits to the daily SEDs for the period MJD 55266-55276. For clarity reasons, the various emission components have been omitted.

Table A.6 summarizes the parameter values of the six model parameters that were assumed to vary. For completeness, the parameters used in modeling the SED during the non-flaring period MJD 54850-54983 are also listed.

\section{Appendix B. Average $\gamma$-ray and neutrino fluxes in quies- cence}

The average $\gamma$-ray flux during the quiescent period is calculated using eq. (3). The reported errors were calculated as follows. For every point of the light curve in the time window MJD 54850-54983, we created a normal distribution of $N=10^{5}$ random numbers, with mean $\mu=F_{\gamma, i}$ and standard deviation $\sigma=\sigma_{F_{\gamma i}}$, where the latter is the statistical error of the measurement. We then performed $N$ times the integral in eq. (3) using a five-point Newton-Cotes method for values drawn from the normal distributions described above. The distribution of the average flux $F_{\gamma}^{q}$ is shown in Fig. B.11. The vertical lines mark the interval where $68 \%$ of the values lies. These are used to derive the reported errors, namely $F_{\gamma}^{q}=4.199_{-0.165}^{+0.175} \times 10^{-10} \mathrm{erg}$ $\mathrm{cm}^{-2} \mathrm{~s}^{-1}$. A similar procedure is used for the model-predicted quiescent neutrino flux $F_{v}^{q}$, where the values $F_{v, i}$ and their respective errors are calculated using eq. (2).

[1] J. A. Hinton, the HESS Collaboration, The status of the HESS project, NAR 48 (2004) 331-337.

[2] E. Lorenz, The MAGIC Collaboration, Status of the $17 \mathrm{~m}$ MAGIC telescope, NAR 48 (2004) 339-344.

[3] J. Holder, V. A. Acciari, E. Aliu, T. Arlen, M. Beilicke, W. Benbow, S. M. Bradbury, J. H. Buckley, V. Bugaev, Y. Butt, K. L. Byrum, A. Cannon, O. Celik, A. Cesarini, L. Ciupik, Y. C. K. Chow, P. Cogan, P. Colin, W. Cui, M. K. Daniel, T. Ergin, A. D. Falcone, S. J. Fegan, J. P. Finley, G. Finnegan, P. Fortin, L. F. Fortson, A. Furniss, G. H. Gillanders, J. Grube, R. Guenette, G. Gyuk, D. Hanna, E. Hays, D. Horan, C. M. Hui,

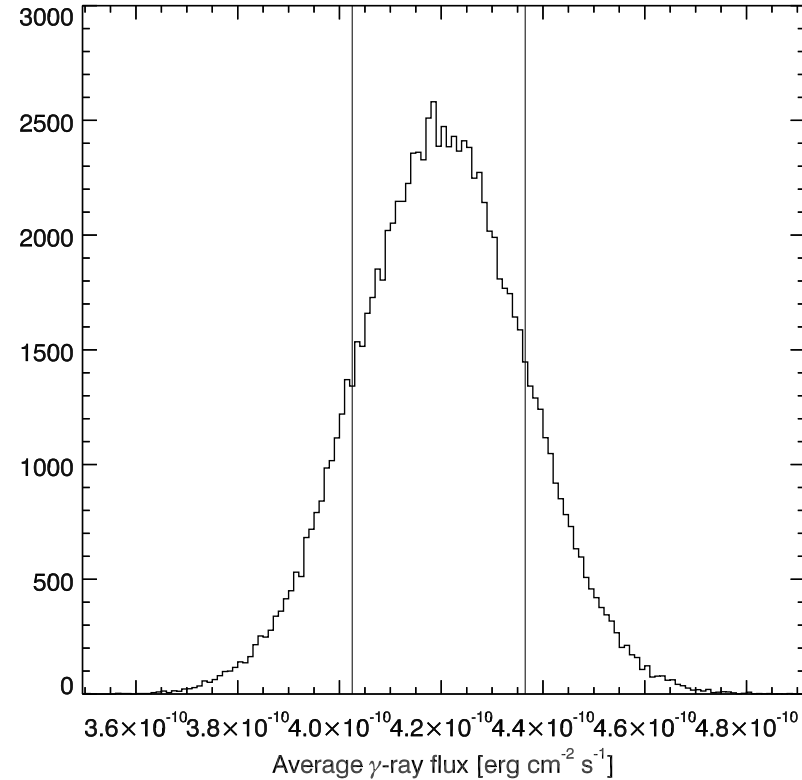

Figure B.11: The distribution of the average $\gamma$-ray flux values during quiescence. The vertical lines mark the interval where $68 \%$ of the values lies.

T. B. Humensky, A. Imran, P. Kaaret, N. Karlsson, M. Kertzman, D. B. Kieda, J. Kildea, A. Konopelko, H. Krawczynski, F. Krennrich, M. J. Lang, S. Lebohec, G. Maier, A. McCann, M. McCutcheon, P. Moriarty, R. Mukherjee, T. Nagai, J. Niemiec, R. A. Ong, D. Pandel, J. S. Perkins, M. Pohl, J. Quinn, K. Ragan, L. C. Reyes, P. T. Reynolds, H. J. Rose, M. Schroedter, G. H. Sembroski, A. W. Smith, D. Steele, S. P. Swordy, J. A. Toner, L. Valcarcel, V. V. Vassiliev, R. Wagner, S. P. Wakely, J. E. Ward, T. C. Weekes, A. Weinstein, R. J. White, D. A. Williams, S. A. Wissel, M. Wood, B. Zitzer, Status of the VERITAS Observatory, in: F. A. Aharonian, W. Hofmann, F. Rieger (Eds.), American Institute of Physics Conference Series, volume 1085 of American Institute of Physics Conference Series, pp. 657-660.

[4] W. B. Atwood, A. A. Abdo, M. Ackermann, W. Althouse, B. Anderson, M. Axelsson, L. Baldini, J. Ballet, D. L. Band, G. Barbiellini, et al., The Large Area Telescope on the Fermi Gamma-Ray Space Telescope Mission, Astrophysical Journal 697 (2009) 1071-1102.

[5] A. P. Marscher, W. K. Gear, Models for high-frequency radio outbursts in extragalactic sources, with application to the early 1983 millimeter-toinfrared flare of 3C 273, Astrophysical Journal 298 (1985) 114-127.

[6] D. Kazanas, D. C. Ellison, The central engine of quasars and active galactic nuclei Hadronic interactions of shock-accelerated relativistic protons, Astrophysical Journal 304 (1986) 178-187.

[7] D. Giannios, UHECRs from magnetic reconnection in relativistic jets, Monthly Notices of the Royal Astronomical Society 408 (2010) L46L50.

[8] D. Giannios, Reconnection-driven plasmoids in blazars: fast flares on a slow envelope, Monthly Notices of the Royal Astronomical Society 431 (2013) 355-363

[9] L. Sironi, A. Spitkovsky, Relativistic Reconnection: An Efficient Source of Non-thermal Particles, Astrophysical Journal Letters 783 (2014) L21.

[10] M. Boettcher, Models for the Spectral Energy Distributions and Variability of Blazars, ArXiv e-prints (2010).

[11] M. Boettcher, Modeling the Spectral Energy Distributions and Variability of Blazars, ArXiv e-prints (2012)

[12] M.-H. Ulrich, L. Maraschi, C. M. Urry, Variability of Active Galactic Nuclei, Ann. Rev. Astron. Asrophys. 35 (1997) 445-502.

[13] G. Fossati, L. Maraschi, A. Celotti, A. Comastri, G. Ghisellini, A unifying 

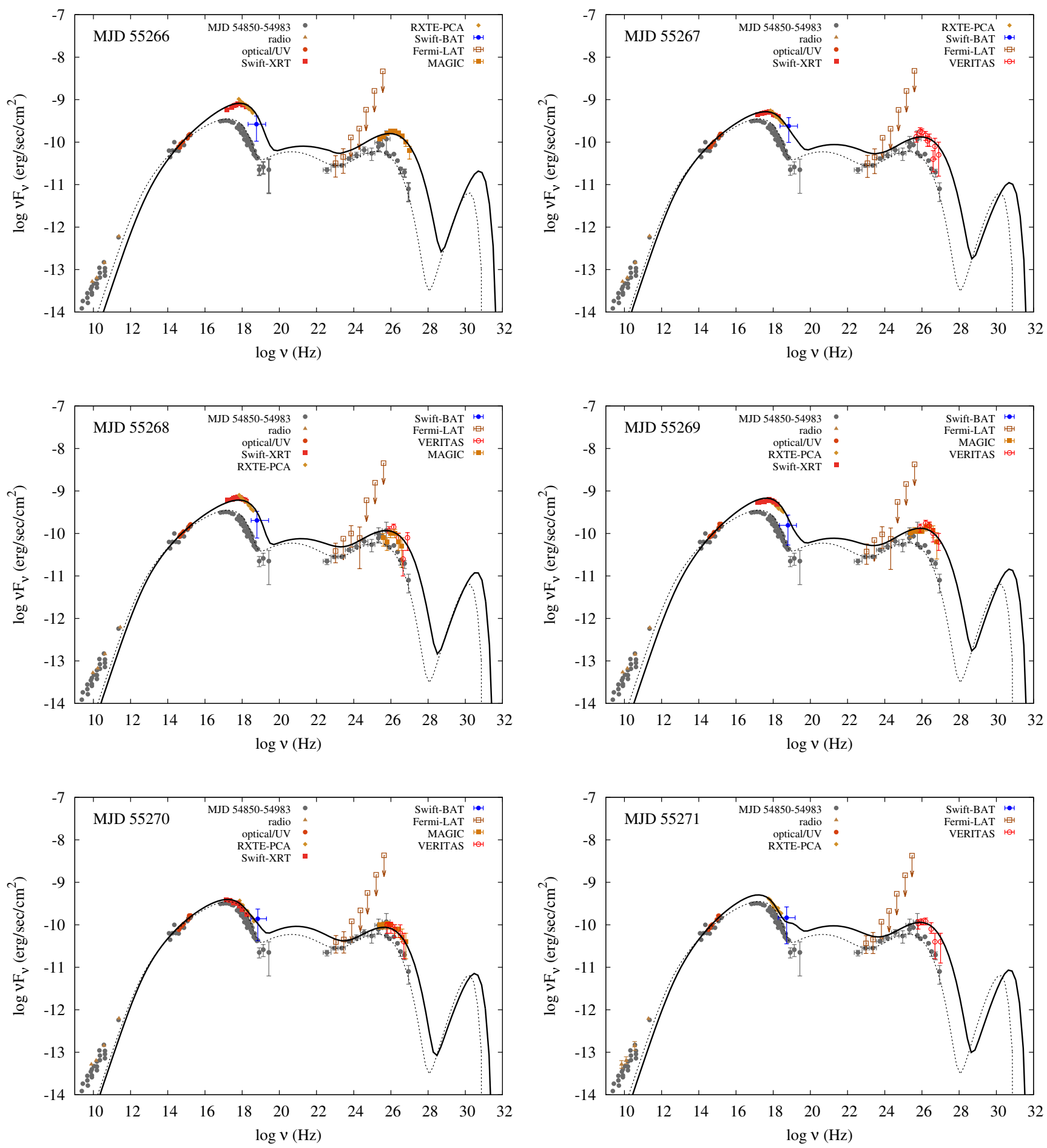

Figure A.10: Model SEDs of Mrk 421 for MJD 55266-55276. All (colored) data-points are from [50]. The grey circles depict the time-averaged SED of Mrk 421 over the period MJD 54850-54983 [47]. This is a good representation of the blazar non-flaring (quiescent) emission. The model-derived spectra that fit the daily SEDs are plotted with black thick lines. The black dotted line is an indicative fit to the quiescent emission. 

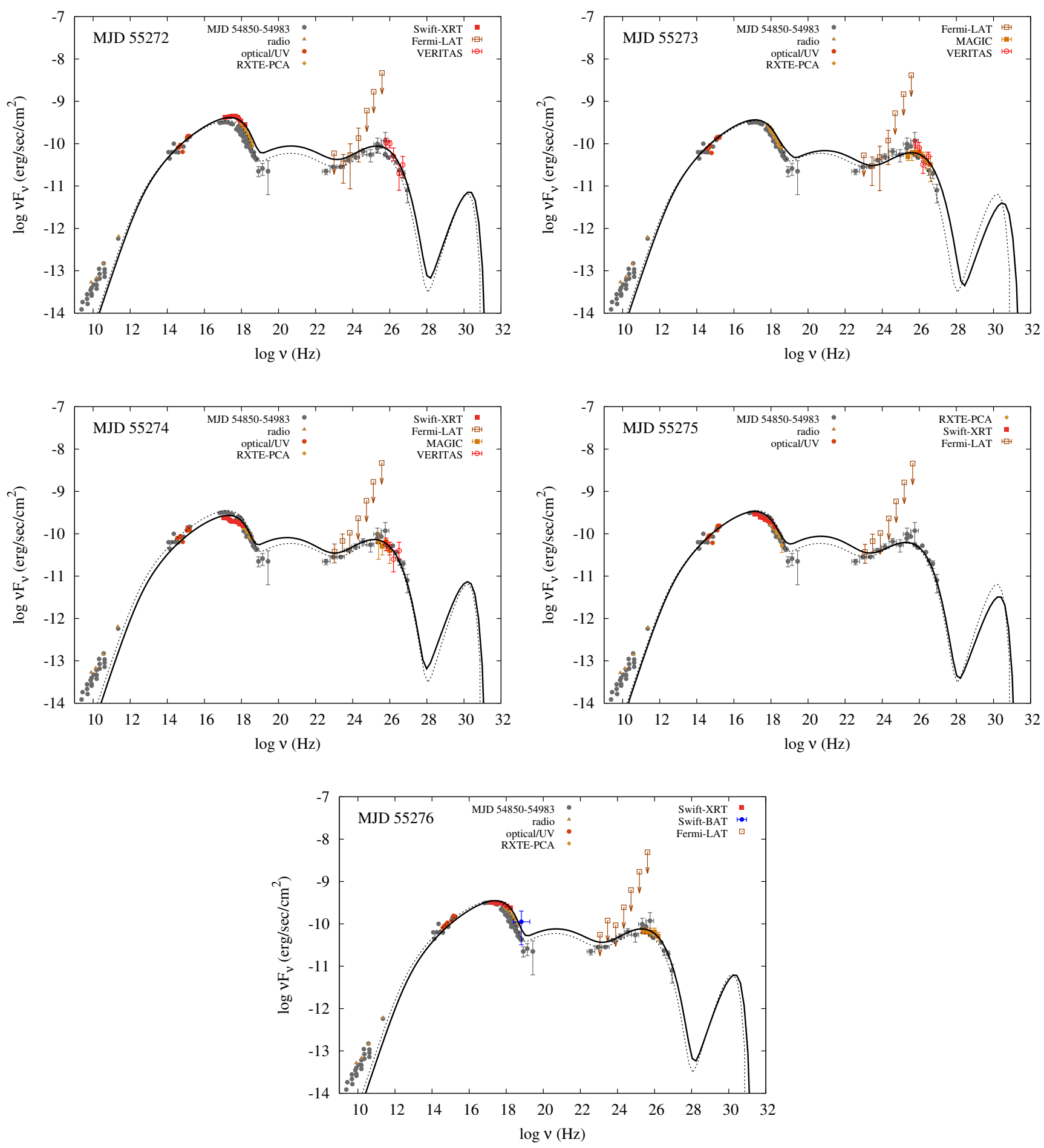

Figure A.10: Model SEDs continued. 
Table A.6: Values of the parameters that were allowed to vary while fitting the SEDs from MJD 55265 to MJD 55277. For reference, the parameter values used for the time-averaged 2009 data [47] are also listed.

\begin{tabular}{ccccccc}
\hline $\begin{array}{c}\text { Date } \\
(\text { MJD })\end{array}$ & $\gamma_{\mathrm{e}, \max }$ & $\ell_{\mathrm{e}}^{\mathrm{inj}}$ & $\gamma_{\mathrm{p}, \max }$ & $\ell_{\mathrm{p}}^{\mathrm{inj}}$ & $\delta$ & $s_{\mathrm{e}}$ \\
\hline 55265 & $1.2 \times 10^{5}$ & $2 \times 10^{-5}$ & $8 \times 10^{6}$ & $3.2 \times 10^{-4}$ & 22.3 & 1.2 \\
55266 & $10^{5}$ & $2 \times 10^{-5}$ & $6.3 \times 10^{6}$ & $4 \times 10^{-4}$ & 23 & 1.0 \\
55267 & $8 \times 10^{4}$ & $1.6 \times 10^{-5}$ & $6.3 \times 10^{6}$ & $5 \times 10^{-4}$ & 22.3 & 1.2 \\
55268 & $10^{5}$ & $2 \times 10^{-5}$ & $4 \times 10^{6}$ & $5 \times 10^{-4}$ & 23.1 & 1.0 \\
55269 & $8 \times 10^{4}$ & $2 \times 10^{-5}$ & $6.3 \times 10^{6}$ & $4 \times 10^{-4}$ & 22 & 1.0 \\
55270 & $5 \times 10^{4}$ & $1.3 \times 10^{-5}$ & $4 \times 10^{6}$ & $8 \times 10^{-4}$ & 22 & 1.2 \\
55271 & $5 \times 10^{4}$ & $2 \times 10^{-5}$ & $6.3 \times 10^{6}$ & $5 \times 10^{-4}$ & 20.5 & 1.0 \\
55272 & $6.3 \times 10^{4}$ & $1.6 \times 10^{-5}$ & $3.2 \times 10^{6}$ & $10^{-3}$ & 20.8 & 1.2 \\
55273 & $5 \times 10^{4}$ & $1.6 \times 10^{-5}$ & $4 \times 10^{6}$ & $6.3 \times 10^{-4}$ & 20.5 & 1.2 \\
55274 & $5 \times 10^{4}$ & $1.1 \times 10^{-5}$ & $2.5 \times 10^{6}$ & $1.6 \times 10^{-3}$ & 20 & 1.2 \\
55275 & $5 \times 10^{4}$ & $2 \times 10^{-5}$ & $3.2 \times 10^{6}$ & $10^{-3}$ & 19 & 1.2 \\
55276 & $6.3 \times 10^{4}$ & $1.6 \times 10^{-5}$ & $3.2 \times 10^{6}$ & $10^{-3}$ & 20 & 1.2 \\
55277 & $5 \times 10^{4}$ & $2 \times 10^{-5}$ & $3.2 \times 10^{6}$ & $10^{-3}$ & 19 & 1.2 \\
\hline $54850-54983^{\mathrm{a}}$ & $6.3 \times 10^{4}$ & $1.3 \times 10^{-5}$ & $3.2 \times 10^{6}$ & $5.6 \times 10^{-4}$ & 21.2 & 1.2 \\
\hline
\end{tabular}

${ }^{\text {a }}$ For the 2009 data, a flatter proton distribution with $s_{\mathrm{p}}=0.6$ was adopted.

view of the spectral energy distributions of blazars, Monthly Notices of the Royal Astronomical Society 299 (1998) 433-448.

14] A. Mücke, R. J. Protheroe, A proton synchrotron blazar model for flaring in Markarian 501, Astroparticle Physics 15 (2001) 121-136.

[15] F. A. Aharonian, TeV gamma rays from BL Lac objects due to synchrotron radiation of extremely high energy protons, New Astron. 5 (2000) 377-395.

[16] A. Mücke, R. J. Protheroe, R. Engel, J. P. Rachen, T. Stanev, BL Lac objects in the synchrotron proton blazar model, Astroparticle Physics 18 (2003) 593-613.

[17] K. Mannheim, $\gamma$ rays and neutrinos from a powerful cosmic accelerator, Physical Review D 48 (1993) 2408-2414.

[18] K. Mannheim, P. L. Biermann, Gamma-ray flaring of 3C 279 - A protoninitiated cascade in the jet?, Astronomy \& Astrophysics 253 (1992) L21L24.

[19] S. Dimitrakoudis, M. Petropoulou, A. Mastichiadis, Self-consistent neutrino and UHE cosmic ray spectra from Mrk 421, Astroparticle Physics 54 (2014) 61-66.

[20] M. Cerruti, A. Zech, C. Boisson, S. Inoue, A hadronic origin for ultrahigh-frequency-peaked BL Lac objects, Monthly Notices of the Royal Astronomical Society 448 (2015) 910-927.

[21] M. Böttcher, A. Reimer, K. Sweeney, A. Prakash, Leptonic and Hadronic Modeling of Fermi-detected Blazars, Astrophysical Journal 768 (2013) 54.

[22] A. Mastichiadis, M. Petropoulou, S. Dimitrakoudis, Mrk 421 as a case study for $\mathrm{TeV}$ and $\mathrm{X}$-ray variability in leptohadronic models, Monthly Notices of the Royal Astronomical Society 434 (2013) 2684-2695.

[23] M. Weidinger, F. Spanier, A self-consistent and time-dependent hybrid blazar emission model. Properties and application, Astronomy \& Astrophysics 573 (2015) A7.

[24] C. Diltz, M. Böttcher, G. Fossati, Time Dependent Hadronic Modeling of Flat Spectrum Radio Quasars, Astrophysical Journal 802 (2015) 133.

[25] M. Petropoulou, S. Dimitrakoudis, P. Padovani, A. Mastichiadis, E. Resconi, Photohadronic origin of $\gamma$-ray BL Lac emission: implications for IceCube neutrinos, Monthly Notices of the Royal Astronomical Society 448 (2015) 2412-2429.

[26] P. G. Tinyakov, I. I. Tkachev, BL Lacertae are Probable Sources of the Observed Ultrahigh Energy Cosmic Rays, Soviet Journal of Experimental and Theoretical Physics Letters 74 (2001) 445-448.

[27] M. R. George, A. C. Fabian, W. H. Baumgartner, R. F. Mushotzky, J. Tueller, On active galactic nuclei as sources of ultra-high energy cosmic rays, Monthly Notices of the Royal Astronomical Society 388 (2008) L59-L63.
[28] Pierre Auger Collaboration, J. Abraham, P. Abreu, M. Aglietta, C. Aguirre, D. Allard, I. Allekotte, J. Allen, P. Allison, J. Alvarez-Muñiz, et al., Correlation of the highest-energy cosmic rays with the positions of nearby active galactic nuclei, Astroparticle Physics 29 (2008) 188-204.

[29] C. Macolino, Pierre Auger Collaboration, Anisotropy studies with the Pierre Auger Observatory, Journal of Physics Conference Series 375 (2012) 052002 .

[30] A. Aab, P. Abreu, M. Aglietta, E. J. Ahn, I. A. Samarai, I. F. M. Albuquerque, I. Allekotte, J. Allen, P. Allison, A. Almela, et al., Searches for Anisotropies in the Arrival Directions of the Highest Energy Cosmic Rays Detected by the Pierre Auger Observatory, Astrophysical Journal 804 (2015) 15.

[31] F. W. Stecker, C. Done, M. H. Salamon, P. Sommers, High-energy neutrinos from active galactic nuclei, Physical Review Letters 66 (1991) 2697-2700.

[32] F. Halzen, E. Zas, Neutrino Fluxes from Active Galaxies: A ModelIndependent Estimate, Astrophysical Journal 488 (1997) 669-674.

[33] A. Atoyan, C. D. Dermer, High-Energy Neutrinos from Photomeson Processes in Blazars, Physical Review Letters 87 (2001) 221102.

[34] K. Mannheim, T. Stanev, P. L. Biermann, Neutrinos from flat-spectrum radio quasars, Astronomy \& Astrophysics 260 (1992) L1-L3.

[35] M. Böttcher, A Hadronic Synchrotron Mirror Model for the "Orphan" TeV Flare in 1ES 1959+650, Astrophysical Journal 621 (2005) 176-180.

[36] F. Halzen, D. Hooper, High energy neutrinos from the TeV Blazar 1ES $1959+650$, Astroparticle Physics 23 (2005) 537-542.

[37] A. Reimer, M. Böttcher, S. Postnikov, Neutrino Emission in the Hadronic Synchrotron Mirror Model: The "Orphan" TeV Flare from 1ES 1959+650, Astrophysical Journal 630 (2005) 186-190.

[38] C. D. Dermer, E. Ramirez-Ruiz, T. Le, Correlation of Photon and Neutrino Fluxes in Blazars and Gamma-Ray Bursts, Astrophysical Journal Letters 664 (2007) L67-L70.

[39] J. P. Rachen, P. Mészáros, Photohadronic neutrinos from transients in astrophysical sources, Physical Review D 58 (1998) 123005.

[40] M. Doert, J. K. Becker, F. Halzen, A. O’Murchadha, W. Rhode, Correlated neutrino and gamma-ray emission from Active Galactic Nuclei - an estimation, Journal of Physics Conference Series 355 (2012) 012039.

[41] N. Fraija, A. Marinelli, TeV $\gamma$-ray fluxes from the long campaigns on Mrk 421 as constraints on the emission of TeV-PeV neutrinos and UHECRs, Astroparticle Physics 70 (2015) 54-61.

[42] IceCube Collaboration, Evidence for High-Energy Extraterrestrial Neutrinos at the IceCube Detector, Science 342 (2013).

[43] M. G. Aartsen, M. Ackermann, J. Adams, J. A. Aguilar, M. Ahlers, M. Ahrens, D. Altmann, T. Anderson, C. Arguelles, T. C. Arlen, et al., 
Observation of High-Energy Astrophysical Neutrinos in Three Years of IceCube Data, Physical Review Letters 113 (2014) 101101.

[44] S. Schoenen, L. Raedel, Detection of a multi-PeV neutrino-induced muon event from the Northern sky with IceCube, The Astronomer's Telegram 7856 (2015) 1.

[45] G. de Vaucouleurs, A. de Vaucouleurs, H. G. Corwin, Jr., R. J. Buta, G. Paturel, P. Fouqué, Third Reference Catalogue of Bright Galaxies. Volume I: Explanations and references. Volume II: Data for galaxies between $0^{h}$ and $12^{h}$. Volume III: Data for galaxies between $12^{h}$ and $24^{h} ., 1991$.

[46] G. D. Şentürk, M. Errando, M. Böttcher, R. Mukherjee, Gamma-ray Observational Properties of TeV-detected Blazars, Astrophysical Journal 764 (2013) 119.

[47] A. A. Abdo, M. Ackermann, M. Ajello, L. Baldini, J. Ballet, G. Barbiellini, D. Bastieri, K. Bechtol, R. Bellazzini, B. Berenji, et al., Fermi Large Area Telescope Observations of Markarian 421: The Missing Piece of its Spectral Energy Distribution, Astrophysical Journal 736 (2011) 131.

[48] S. Dimitrakoudis, A. Mastichiadis, R. J. Protheroe, A. Reimer, The timedependent one-zone hadronic model. First principles, Astronomy \& Astrophysics 546 (2012) A120.

[49] P. Padovani, M. Petropoulou, P. Giommi, E. Resconi, A simplified view of blazars: the neutrino background, Monthly Notices of the Royal Astronomical Society 452 (2015) 1877-1887.

[50] J. e. a. Aleksić, Unprecedented study of the broadband emission of Mrk 421 during flaring activity in March 2010, Astronomy \& Astrophysics 578 (2015) A22.

[51] M. G. Aartsen, et al., Search for a diffuse flux of astrophysical muon neutrinos with the IceCube 59-string configuration, Phys. Rev. D89 (2014) 062007.

[52] M. G. Aartsen, et al., Evidence for astrophysical muon neutrinos from the northern sky with icecube, Phys. Rev. Lett. 115 (2015) 081102.

[53] M. G. Aartsen, M. Ackermann, J. Adams, J. A. Aguilar, M. Ahlers, M. Ahrens, D. Altmann, T. Anderson, C. Arguelles, T. C. Arlen, et al., Searches for Extended and Point-like Neutrino Sources with Four Years of IceCube Data, Astrophysical Journal 796 (2014) 109.

[54] M. G. Aartsen, R. Abbasi, M. Ackermann, J. Adams, J. A. Aguilar, M. Ahlers, D. Altmann, C. Arguelles, J. Auffenberg, X. Bai, et al., Probing the origin of cosmic rays with extremely high energy neutrinos using the IceCube Observatory, Physical Review D 88 (2013) 112008.

[55] K. Mannheim, P. L. Biermann, W. M. Kruells, A novel mechanism for nonthermal X-ray emission, Astronomy \& Astrophysics 251 (1991) 723 731.

[56] F. W. Stecker, Effect of Photomeson Production by the Universal Radiation Field on High-Energy Cosmic Rays, Physical Review Letters 21 (1968) 1016-1018.

[57] A. Mücke, R. Engel, J. P. Rachen, R. J. Protheroe, T. Stanev, Monte Carlo simulations of photohadronic processes in astrophysics, Computer Physics Communications 124 (2000) 290-314.

[58] R. J. Protheroe, P. A. Johnson, Propagation of ultra high energy protons and gamma rays over cosmological distances and implications for topological defect models, Astroparticle Physics 4 (1996) 253-269.

[59] A. Mastichiadis, R. J. Protheroe, J. G. Kirk, Spectral and temporal signatures of ultrarelativistic protons in compact sources. I. Effects of BetheHeitler pair production, Astronomy \& Astrophysics 433 (2005) 765-776.

[60] M. Petropoulou, D. Giannios, S. Dimitrakoudis, Implications of a PeV neutrino spectral cut-off in gamma-ray burst models, Monthly Notices of the Royal Astronomical Society 445 (2014) 570-580.

[61] S. Adrian-Martinez, et al., Searches for Point-like and extended neutrino sources close to the Galactic Centre using the ANTARES neutrino Telescope, Astrophys. J. 786 (2014) L5.

[62] E. Bugaev, T. Montaruli, Y. Shlepin, I. A. Sokalski, Propagation of tau neutrinos and tau leptons through the earth and their detection in underwater / ice neutrino telescopes, Astropart. Phys. 21 (2004) 491-509.

[63] R. Abbasi, et al., Time-Integrated Searches for Point-like Sources of Neutrinos with the 40-String IceCube Detector, Astrophys. J. 732 (2011) 18.

[64] M. Honda, T. Kajita, K. Kasahara, S. Midorikawa, T. Sanuki, Calculation of atmospheric neutrino flux using the interaction model calibrated with atmospheric muon data, Physical Review D 75 (2007) 043006.

[65] M. Petropoulou, A. Mastichiadis, Bethe-Heitler emission in BL Lacs: filling the gap between X-rays and $\gamma$-rays, Monthly Notices of the Royal Astronomical Society 447 (2015) 36-48.

[66] P. Ubertini, F. Lebrun, G. Di Cocco, A. Bazzano, A. J. Bird, K. Broen- stad, A. Goldwurm, G. La Rosa, C. Labanti, P. Laurent, I. F. Mirabel, E. M. Quadrini, B. Ramsey, V. Reglero, L. Sabau, B. Sacco, R. Staubert, L. Vigroux, M. C. Weisskopf, A. A. Zdziarski, IBIS: The Imager on-board INTEGRAL, Astronomy \& Astrophysics 411 (2003) L131-L139.

[67] X. Wu, M. Su, A. Bravar, J. Chang, Y. Fan, M. Pohl, R. Walter, PANGU: A high resolution gamma-ray space telescope, in: Society of PhotoOptical Instrumentation Engineers (SPIE) Conference Series, volume 9144 of Society of Photo-Optical Instrumentation Engineers (SPIE) Conference Series, p. 0.

[68] S. D. Hunter, P. F. Bloser, G. O. Depaola, M. P. Dion, G. A. DeNolfo, A. Hanu, M. Iparraguirre, J. Legere, F. Longo, M. L. McConnell, S. F. Nowicki, J. M. Ryan, S. Son, F. W. Stecker, A pair production telescope for medium-energy gamma-ray polarimetry, Astroparticle Physics 59 (2014) 18-28.

[69] M. Balokovic, A. Furniss, G. Madejski, F. Harrison, NuSTAR detects extreme X-ray flaring of Mrk 421, The Astronomer's Telegram 4974 (2013) 1 .

[70] J. Cortina, J. Holder, MAGIC and VERITAS detect an unprecedented flaring activity from Mrk 421 in very high energy gamma-rays, The Astronomer's Telegram 4976 (2013) 1

[71] T. Hovatta, M. Balokovic, J. L. Richards, W. Max-Moerbeck, A. C. S. Readhead, An increase in the millimeter and centimeter band flux density of Mrk421, The Astronomer's Telegram 5107 (2013) 1.

[72] D. Paneque, F. D'Ammando, M. Orienti, A. Falcon, Fermi-LAT and Swift-XRT observe exceptionally high activity from the nearby $\mathrm{TeV}$ blazar Mrk421, The Astronomer's Telegram 4977 (2013) 1.

[73] V. S. Paliya, M. Böttcher, C. Diltz, C. S. Stalin, S. Sahayanathan, C. D. Ravikumar, The Violent Hard X-Ray Variability of Mrk 421 Observed by NuSTAR in 2013 April, Astrophysical Journal 811 (2015) 143.

[74] J. G. Learned, S. Pakvasa, Detecting $v$ oscillations at PeV energies, Astroparticle Physics 3 (1995) 267-274.

[75] F. D'Ammando, M. Orienti, Fermi LAT detection of a GeV flare from the BL Lac object Mrk 421, The Astronomer's Telegram 4261 (2012) 1.

[76] T. Hovatta, M. Petropoulou, J. L. Richards, D. Giannios, K. Wiik, M. Baloković, A. Lähteenmäki, B. Lott, W. Max-Moerbeck, V. Ramakrishnan, A. C. S. Readhead, A combined radio and GeV $\gamma$-ray view of the 2012 and 2013 flares of Mrk 421, Monthly Notices of the Royal Astronomical Society 448 (2015) 3121-3131.

[77] M. G. Aartsen, M. Ackermann, J. Adams, J. A. Aguilar, M. Ahlers, M. Ahrens, D. Altmann, T. Anderson, M. Archinger, C. Arguelles, et al., Searches for Time-dependent Neutrino Sources with IceCube Data from 2008 to 2012, Astrophysical Journal 807 (2015) 46.

[78] S. Coenders, Results of neutrino point source searches with 2008-2014 IceCube data above $10 \mathrm{TeV}$, To appear in the proceedings of The 34th International Cosmic Ray Conference PoS(ICRC2015)1047 (2015).

[79] K. Murase, Y. Inoue, C. D. Dermer, Diffuse Neutrino Intensity from the Inner Jets of Active Galactic Nuclei: Impacts of External Photon Fields and the Blazar Sequence, ArXiv e-prints (2014).

[80] C. Kopper, W. Giang, N. Kurahashi, Observation of Astrophysical Neutrinos in Four Years of IceCube Data, To appear in the proceedings of The 34th International Cosmic Ray Conference PoS(ICRC2015)1081 (2015).

[81] J. G. Kirk, A. Mastichiadis, Neutrons from active galactic nuclei, Astronomy \& Astrophysics 213 (1989) 75-79.

[82] P. M. Giovanoni, D. Kazanas, Flat-spectrum radio sources - Cosmic conspiracy or relativistic neutrons?, Nature 345 (1990) 319-322.

[83] A. M. Atoyan, C. D. Dermer, Neutral Beams from Blazar Jets, Astrophysical Journal 586 (2003) 79-96.

[84] P. Baerwald, M. Bustamante, W. Winter, UHECR Escape Mechanisms for Protons and Neutrons from Gamma-Ray Bursts, and the Cosmic-RayNeutrino Connection, ApJ 768 (2013) 186.

[85] F. W. Stecker, M. A. Malkan, S. T. Scully, Intergalactic Photon Spectra from the Far-IR to the UV Lyman Limit for $0 ; \mathrm{z} ; 6$ and the Optical Depth of the Universe to High-Energy Gamma Rays, Astrophysical Journal 648 (2006) 774-783.

[86] J. D. Finke, S. Razzaque, C. D. Dermer, Modeling the Extragalactic Background Light from Stars and Dust, Astrophysical Journal 712 (2010) 238-249.

[87] T. M. Kneiske, H. Dole, A lower-limit flux for the extragalactic background light, Astronomy \& Astrophysics 515 (2010) A19.

[88] J. Beringer, J.-F. Arguin, R. M. Barnett, K. Copic, O. Dahl, D. E. Groom, C.-J. Lin, J. Lys, H. Murayama, C. G. Wohl, W.-M. Yao, P. A. 
Zyla, C. Amsler, M. Antonelli, D. M. Asner, H. Baer, H. R. Band, T. Basaglia, C. W. Bauer, J. J. Beatty, V. I. Belousov, E. Bergren, G. Bernardi, W. Bertl, S. Bethke, H. Bichsel, O. Biebel, E. Blucher, S. Blusk, G. Brooijmans, O. Buchmueller, R. N. Cahn, M. Carena, A. Ceccucci, D. Chakraborty, M.-C. Chen, R. S. Chivukula, G. Cowan, G. D’Ambrosio, T. Damour, D. de Florian, A. de Gouvêa, T. DeGrand, P. de Jong, G. Dissertori, B. Dobrescu, M. Doser, M. Drees, D. A. Edwards, S. Eidelman, J. Erler, V. V. Ezhela, W. Fetscher, B. D. Fields, B. Foster, T. K. Gaisser, L. Garren, H.-J. Gerber, G. Gerbier, T. Gherghetta, S. Golwala, M. Goodman, C. Grab, A. V. Gritsan, J.-F. Grivaz, M. Grünewald, A. Gurtu, T. Gutsche, H. E. Haber, K. Hagiwara, C. Hagmann, C. Hanhart, S. Hashimoto, K. G. Hayes, M. Heffner, B. Heltsley, J. J. Hernández-Rey, K. Hikasa, A. Höcker, J. Holder, A. Holtkamp, J. Huston, J. D. Jackson, K. F. Johnson, T. Junk, D. Karlen, D. Kirkby, S. R. Klein, E. Klempt, R. V. Kowalewski, F. Krauss, . Ligeti, T. M. Liss, L. Littenberg, K. S. Lugovsky, S. B. Lugovsky, T. Mannel, A. V. Manohar, W. J. Marciano, A. D. Martin, A. Masoni, J. Matthews, D. Milstead, R. Miquel, K. Mönig, F. Moortgat, K. Nakamura, M. Narain, P. Nason, S. Navas, M. Neubert, P. Nevski, Y. Nir, K. A. Olive, L. Pape, J. Parsons, C. Patrignani, J. A. Peacock, S. T. Petcov, A. Piepke, A. Pomarol, G. Punzi, A. Quadt, S. Raby, G. Raffelt, B. N. Ratcliff, P. Richardson, S. Roesler, S. Rolli, A. Romaniouk, L. J. Rosenberg, J. L. Rosner, C. T. Sachrajda, Y. Sakai, G. P. Salam, S. Sarkar, F. Sauli, O. Schneider, K. Scholberg, D. Scott, W. G. Seligman, M. H. Shaevitz, S. R. Sharpe, M. Silari, T. Sjöstrand, Review of Particle Physics, Physical Review D 86 (2012) 010001.

[89] M. Lemoine, Extragalactic magnetic fields and the second knee in the cosmic-ray spectrum, Physical Review D 71 (2005) 083007.

[90] M. D. Kistler, T. Stanev, H. Yüksel, Cosmic PeV neutrinos and the sources of ultrahigh energy protons, Physical Review D 90 (2014) 123006. 


\section{mrs.pdf}

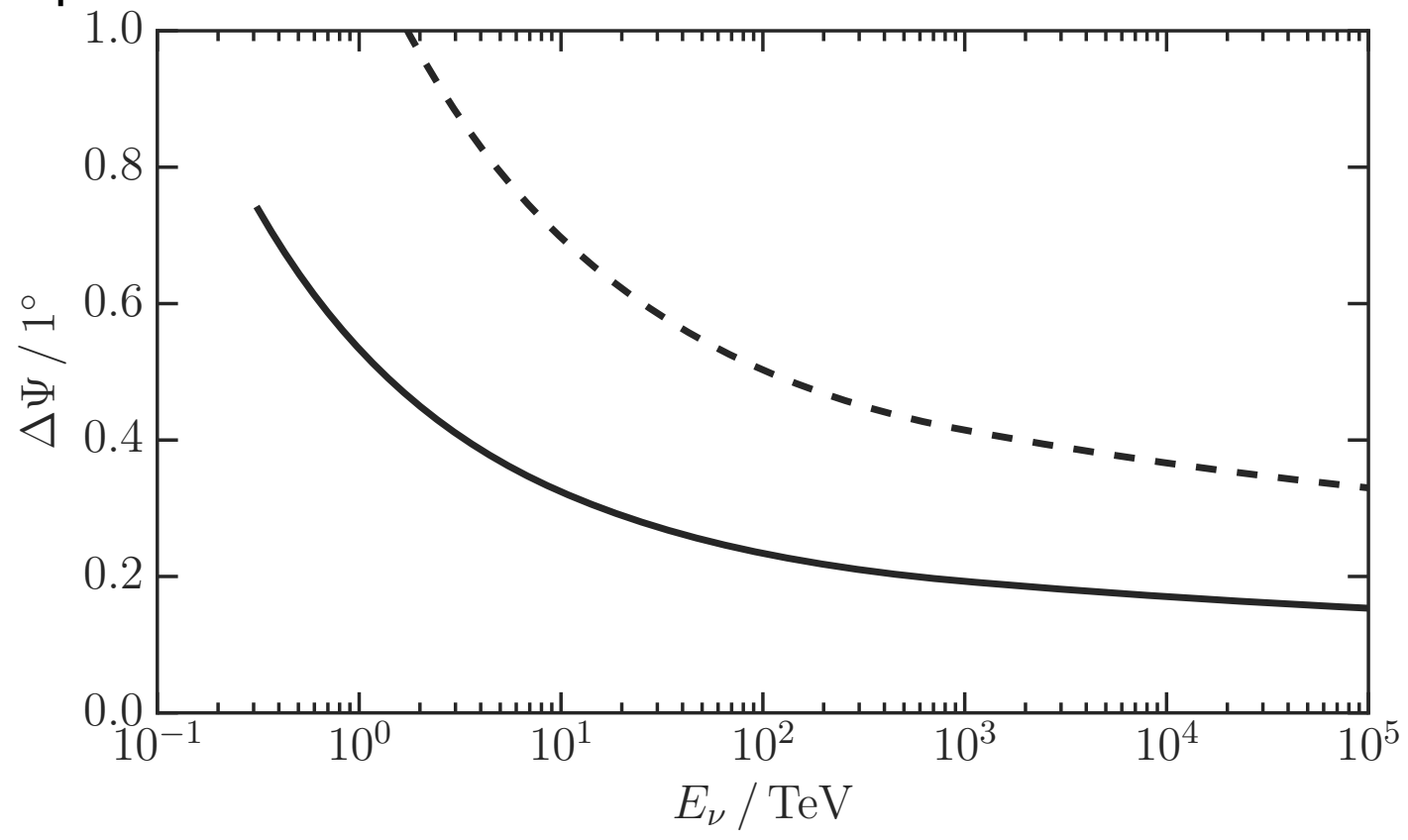




\section{mrk421_mjd55273 vizier.pdf}

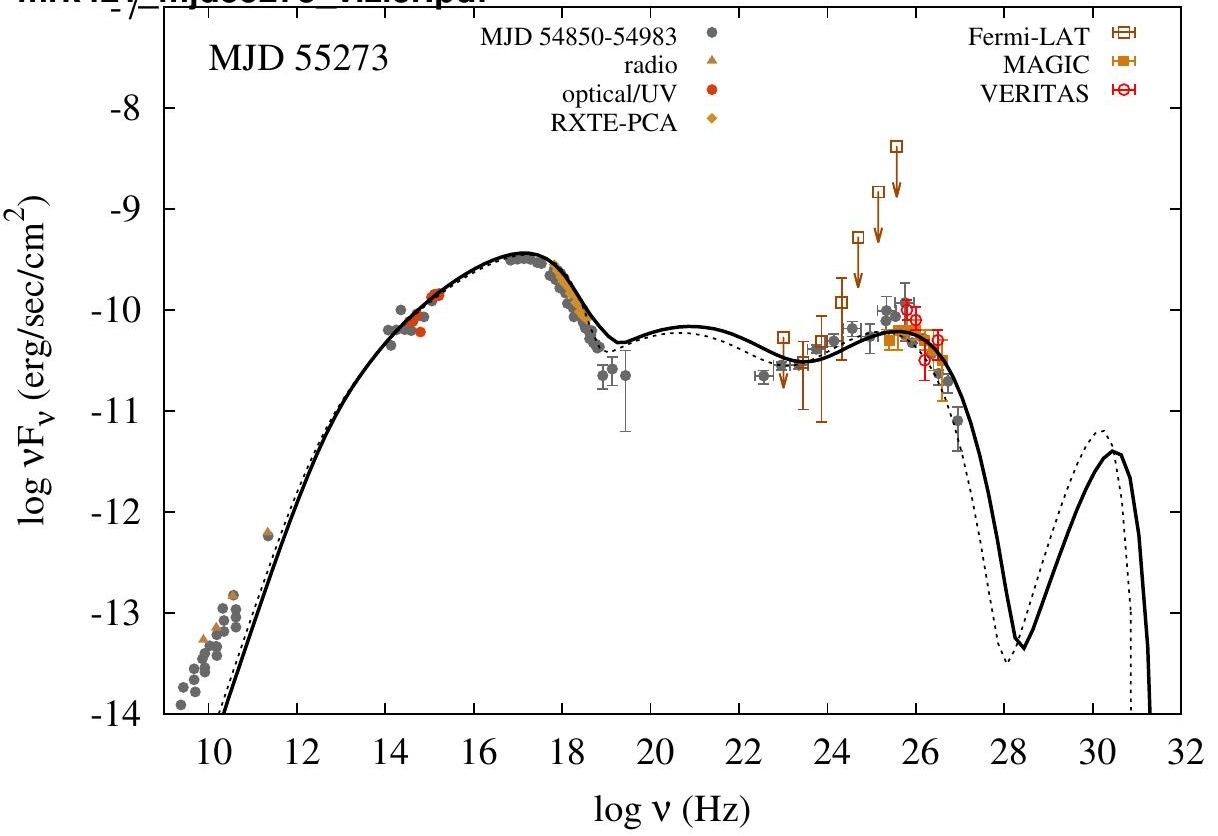




\section{photon_nt lc v1.pdf}

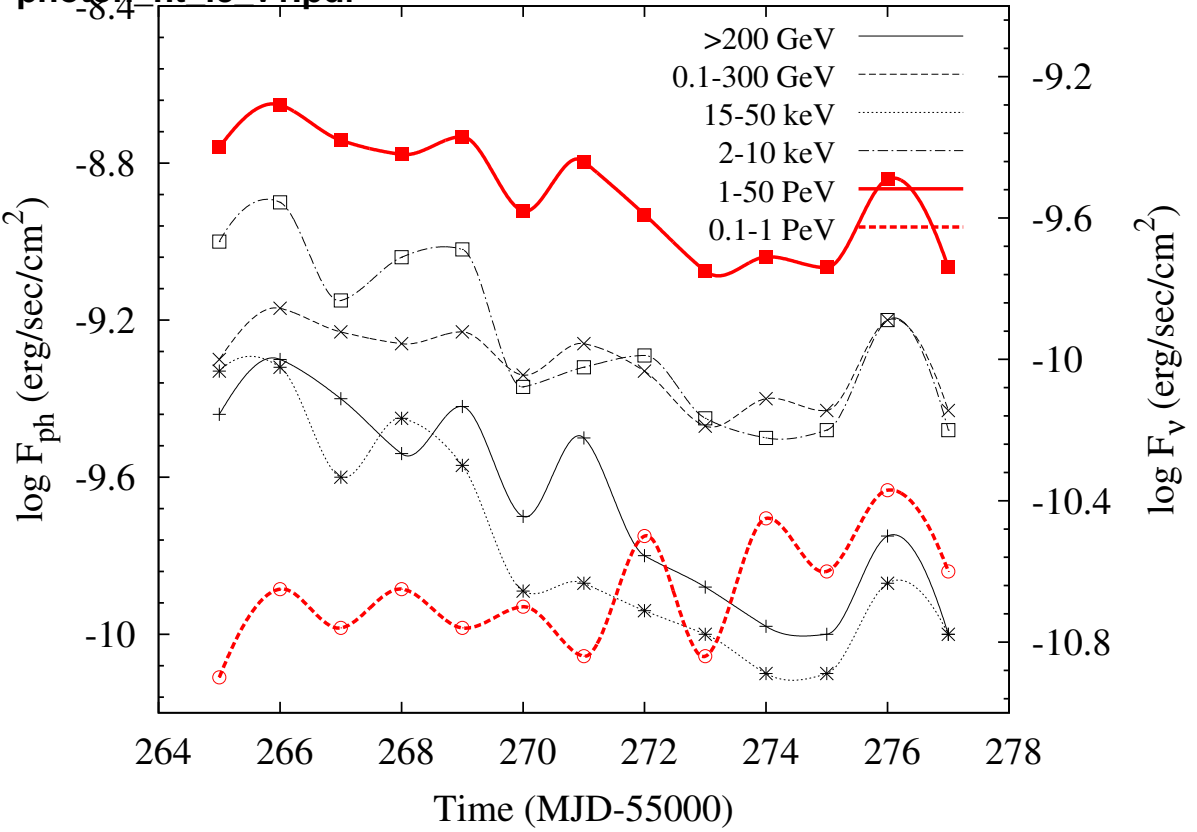




\section{mrk4211_mjd55265 components vizier.pdf}

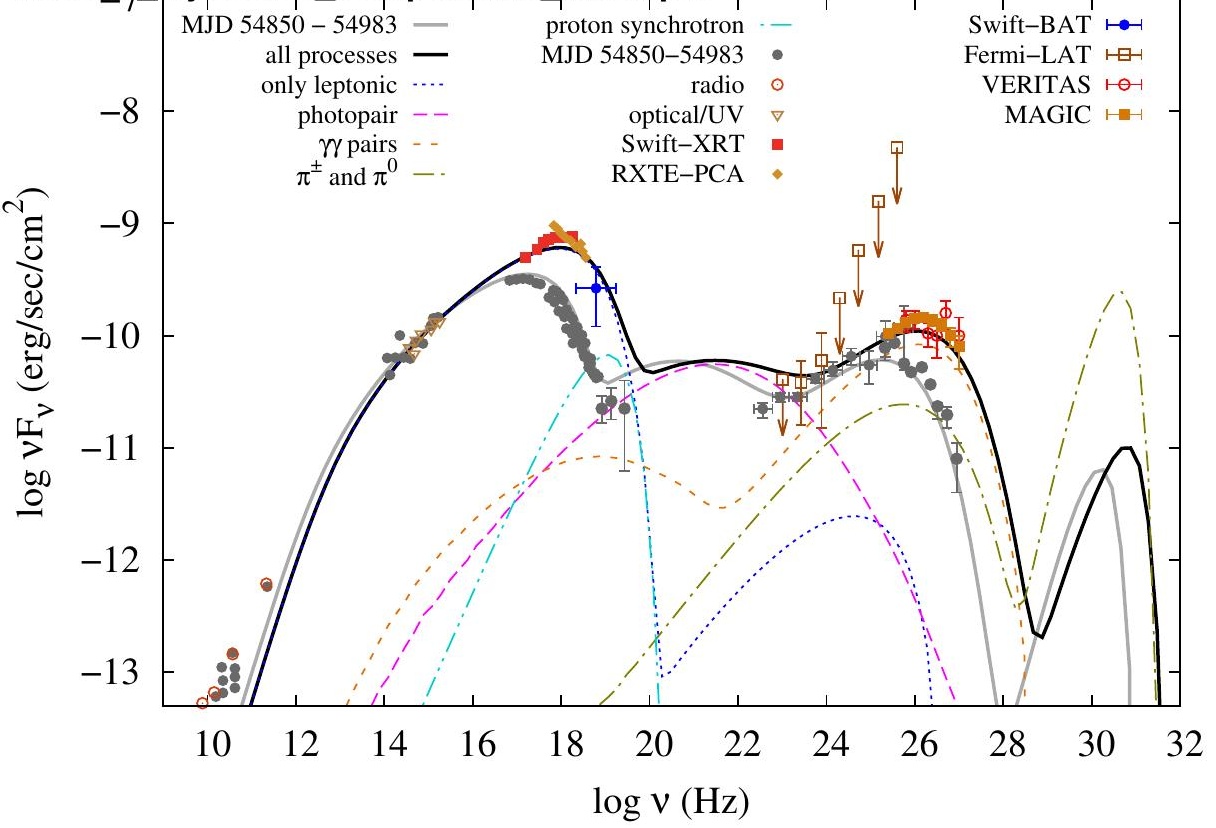




\section{longterm_lightcurve.pdf}
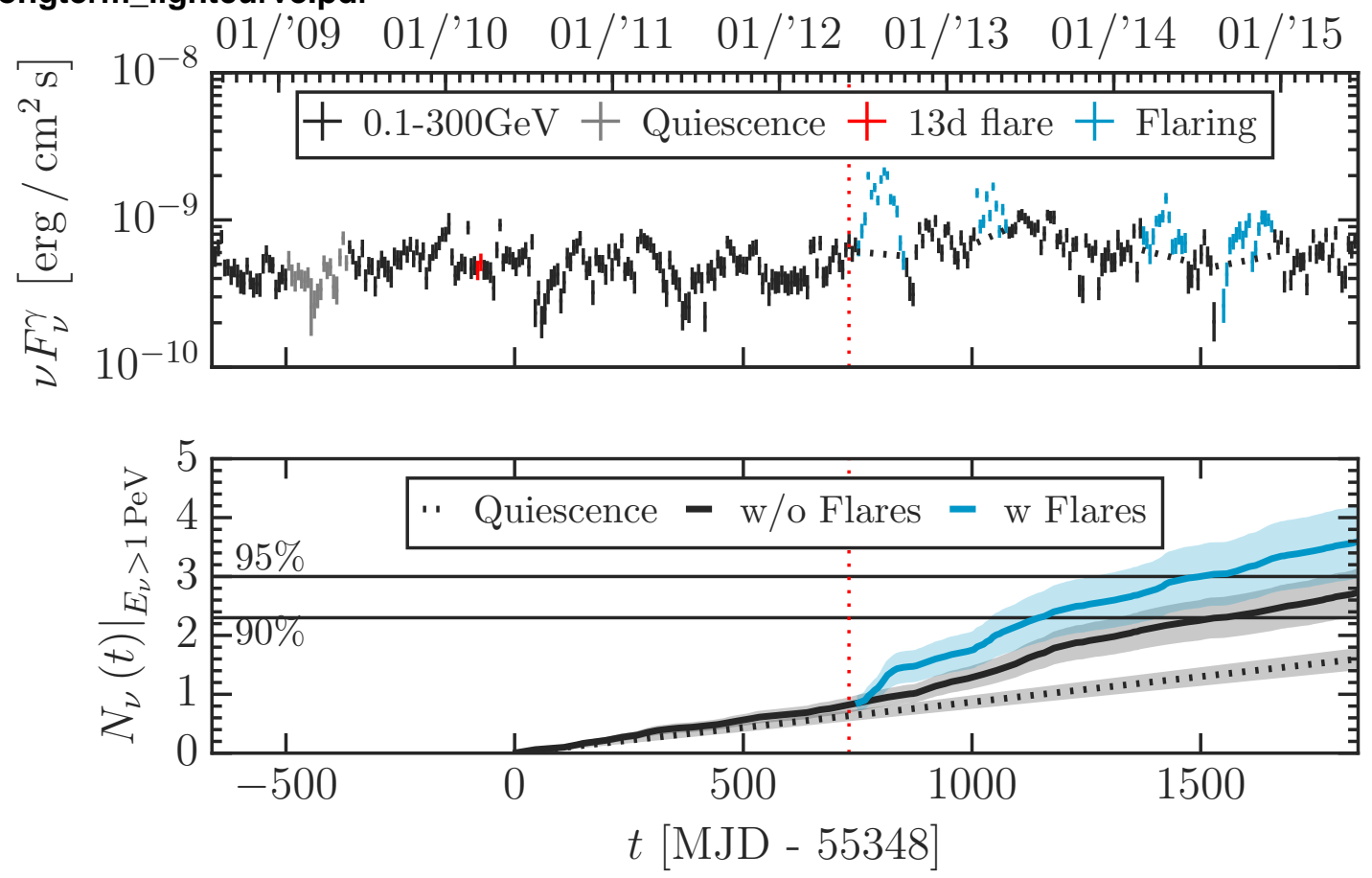


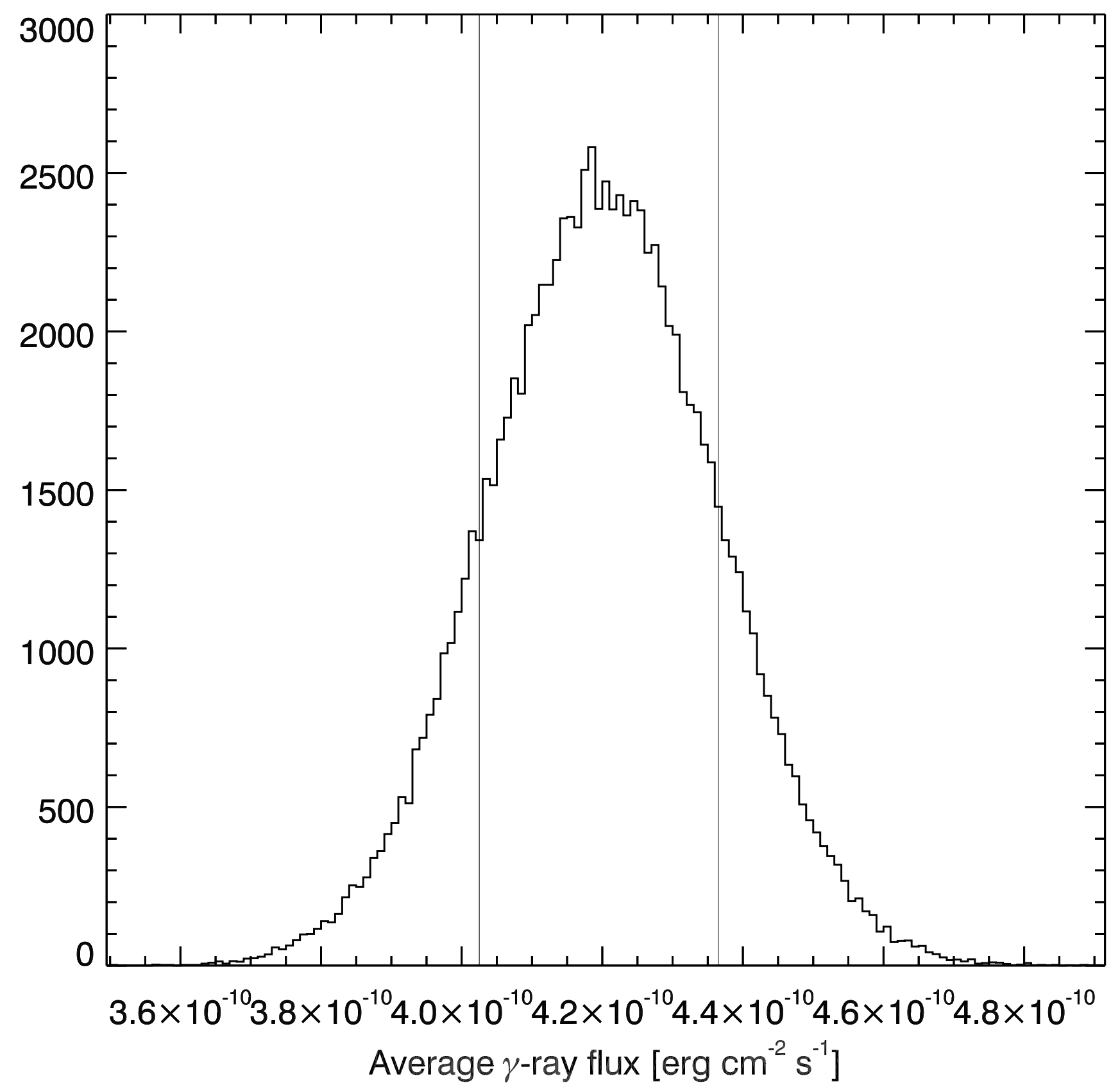




\section{mrk421_mjd55277 components vizier.pdf}

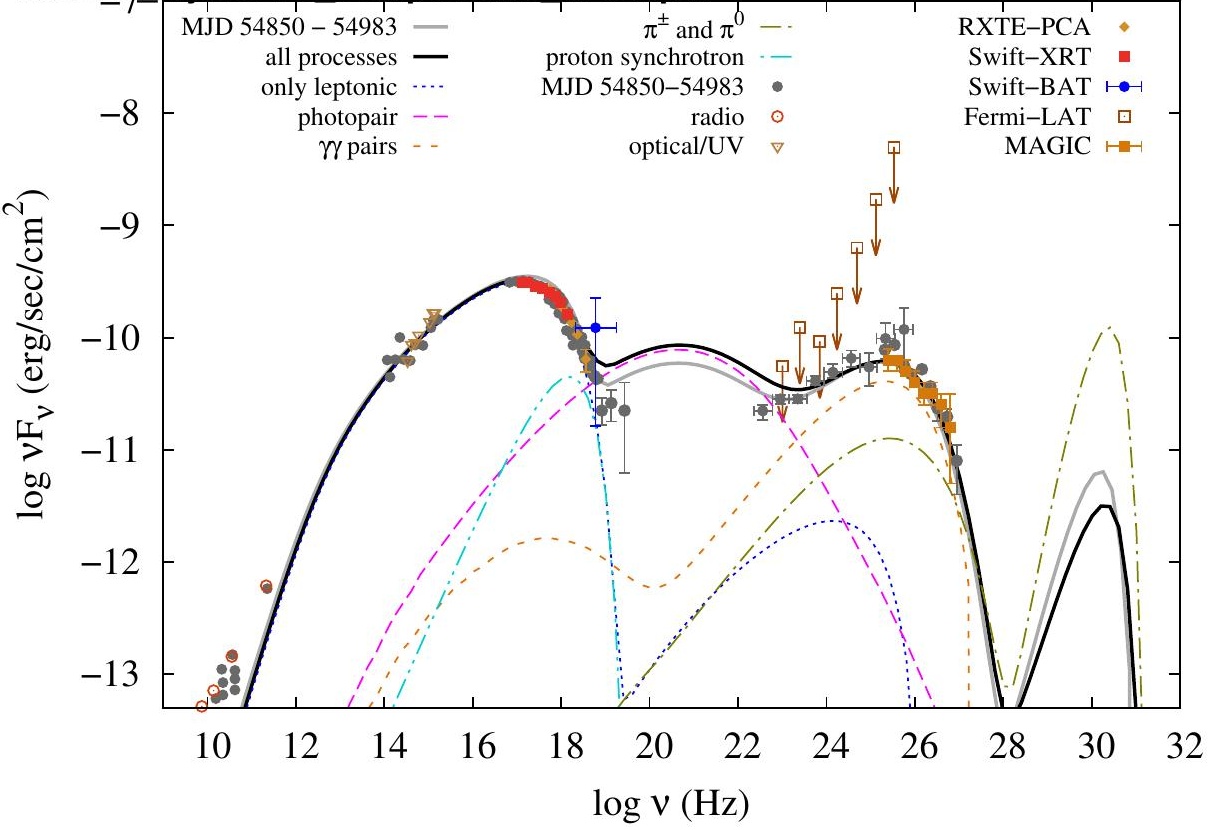




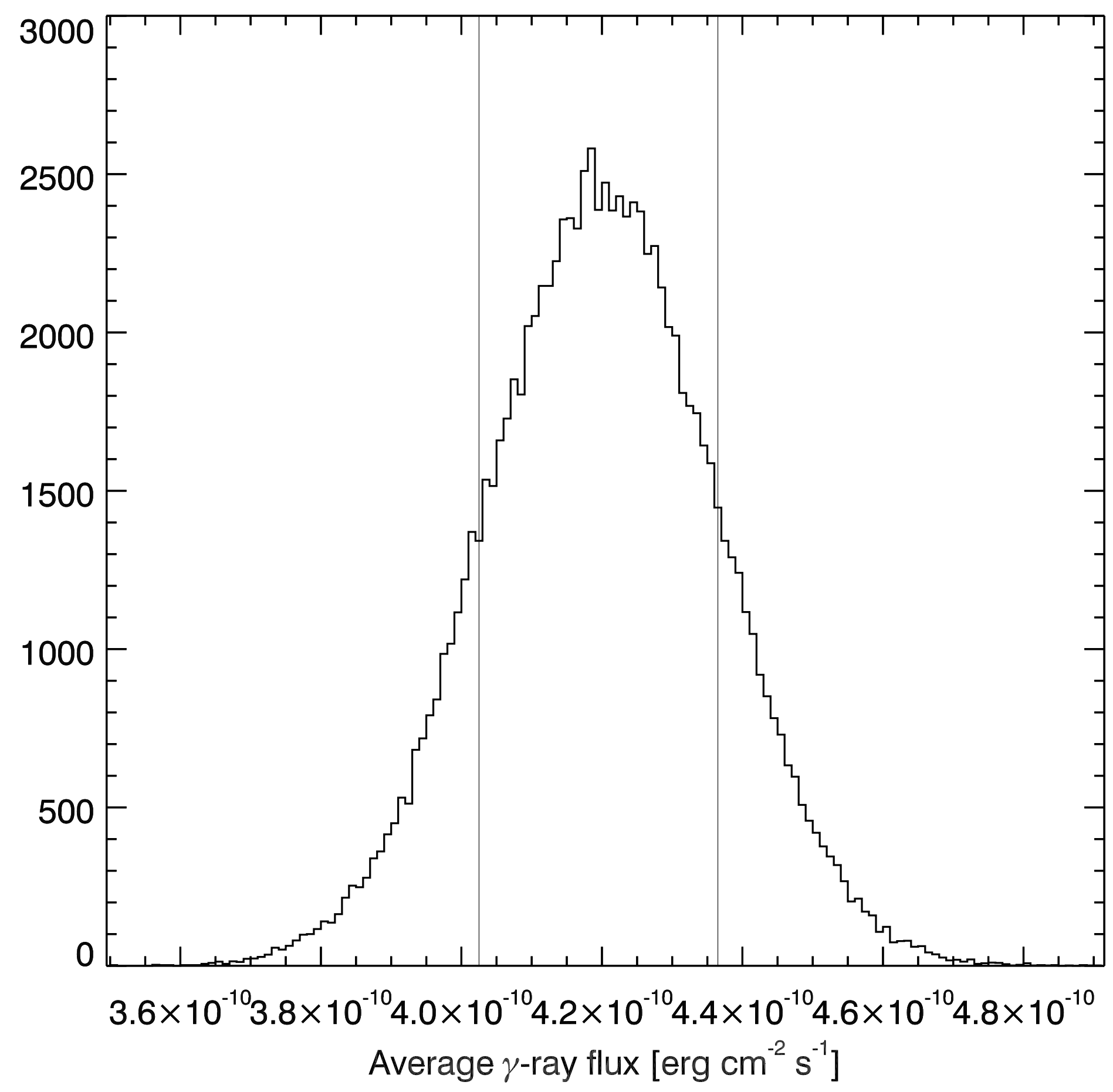




\section{error-contributions.pdf}

$\square A \square A$ w Flares $\square F_{\gamma}^{q}$

$F_{i, \gamma}$

$\square$

$\dot{N}_{\nu}^{q}$

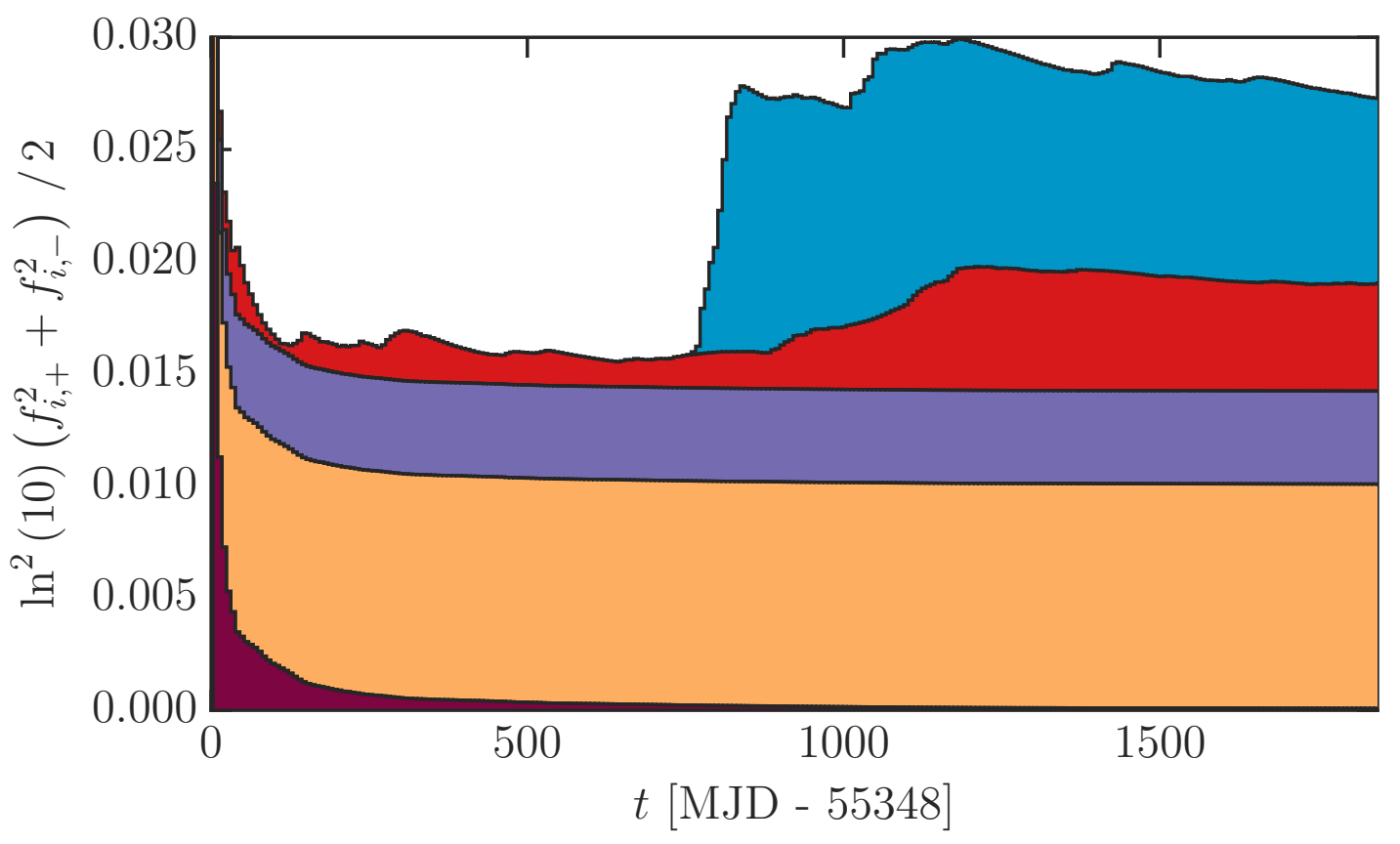




\section{mrk421_mjd55266 vizier.pdf}

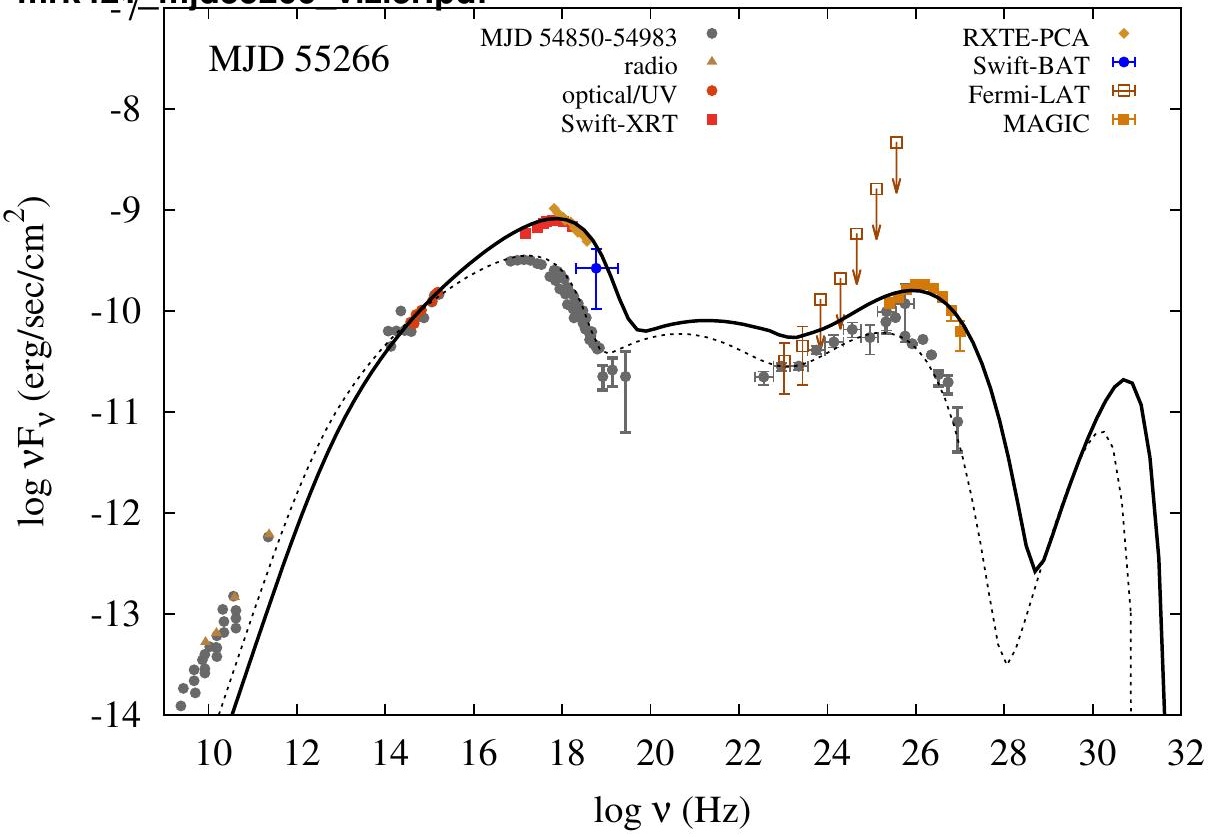




\section{sensitivity.pdf}

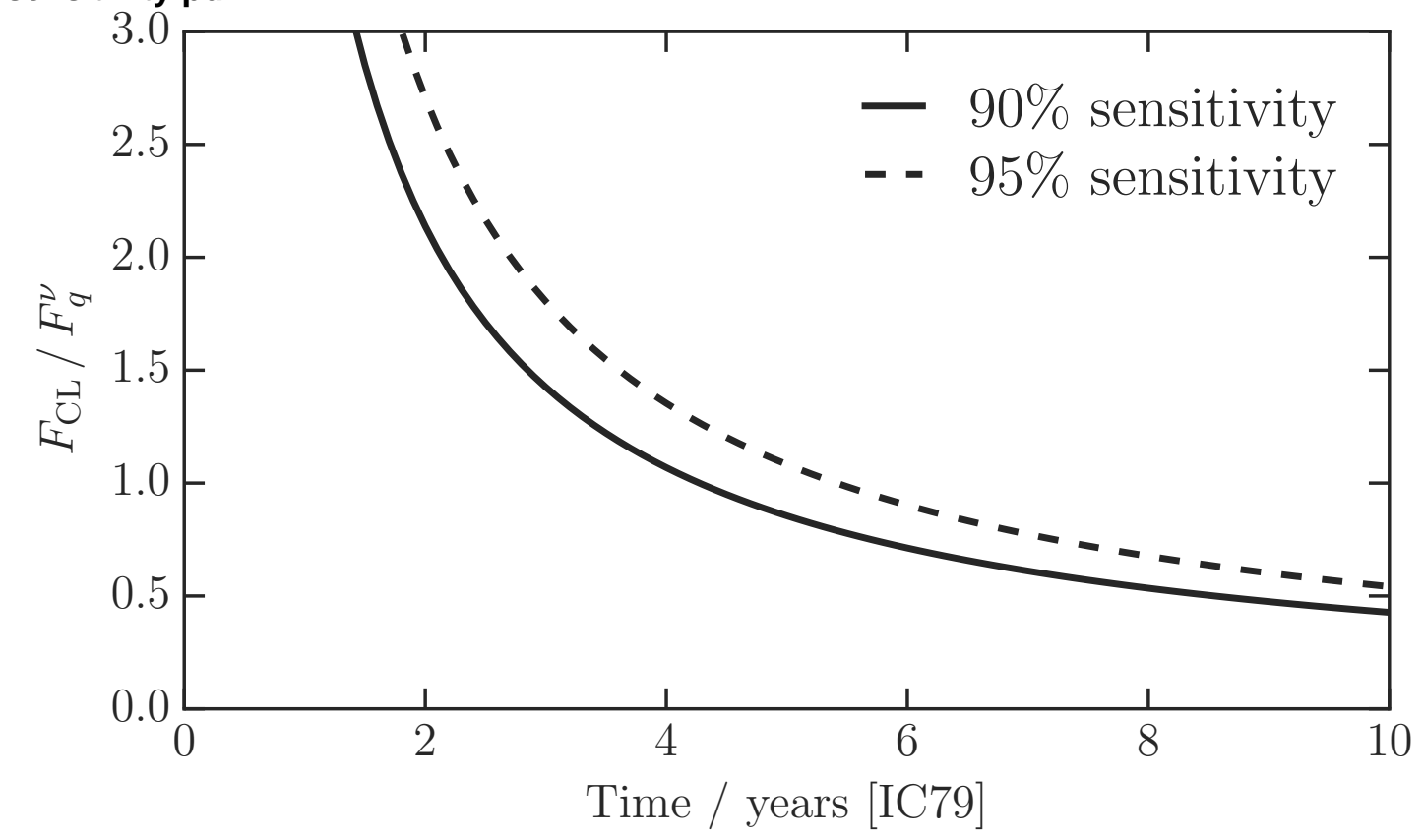




\section{ev_rate.pdf}

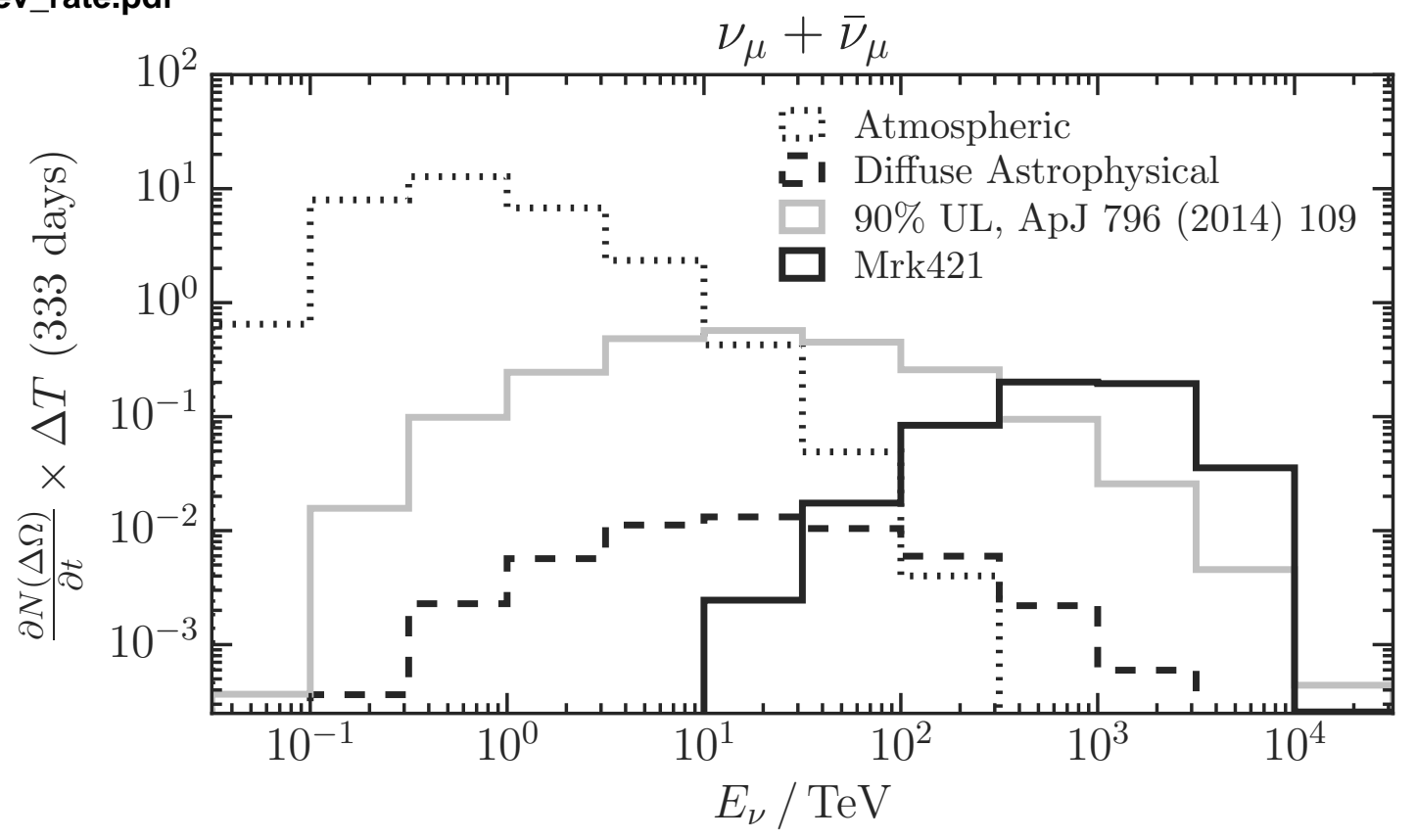




\section{mrk421_mjd55271 vizier.pdf}

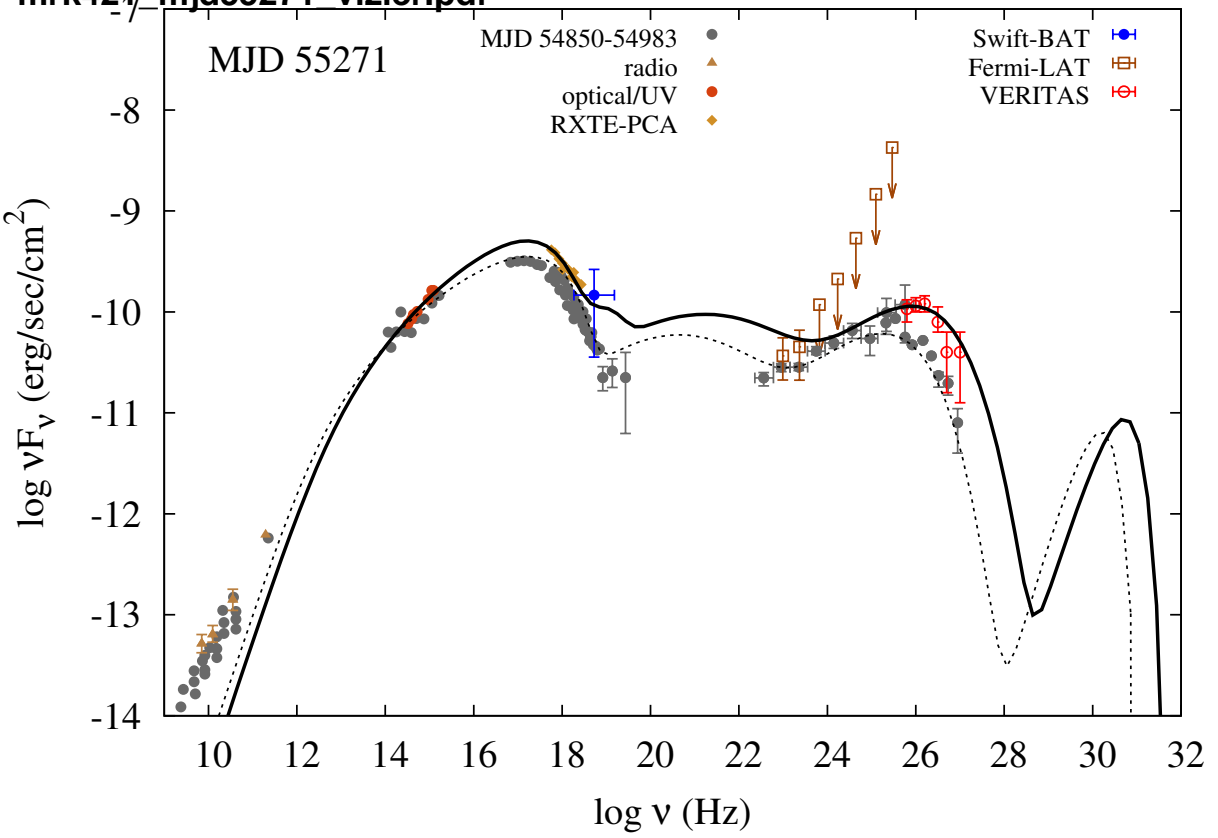




\section{Figure}

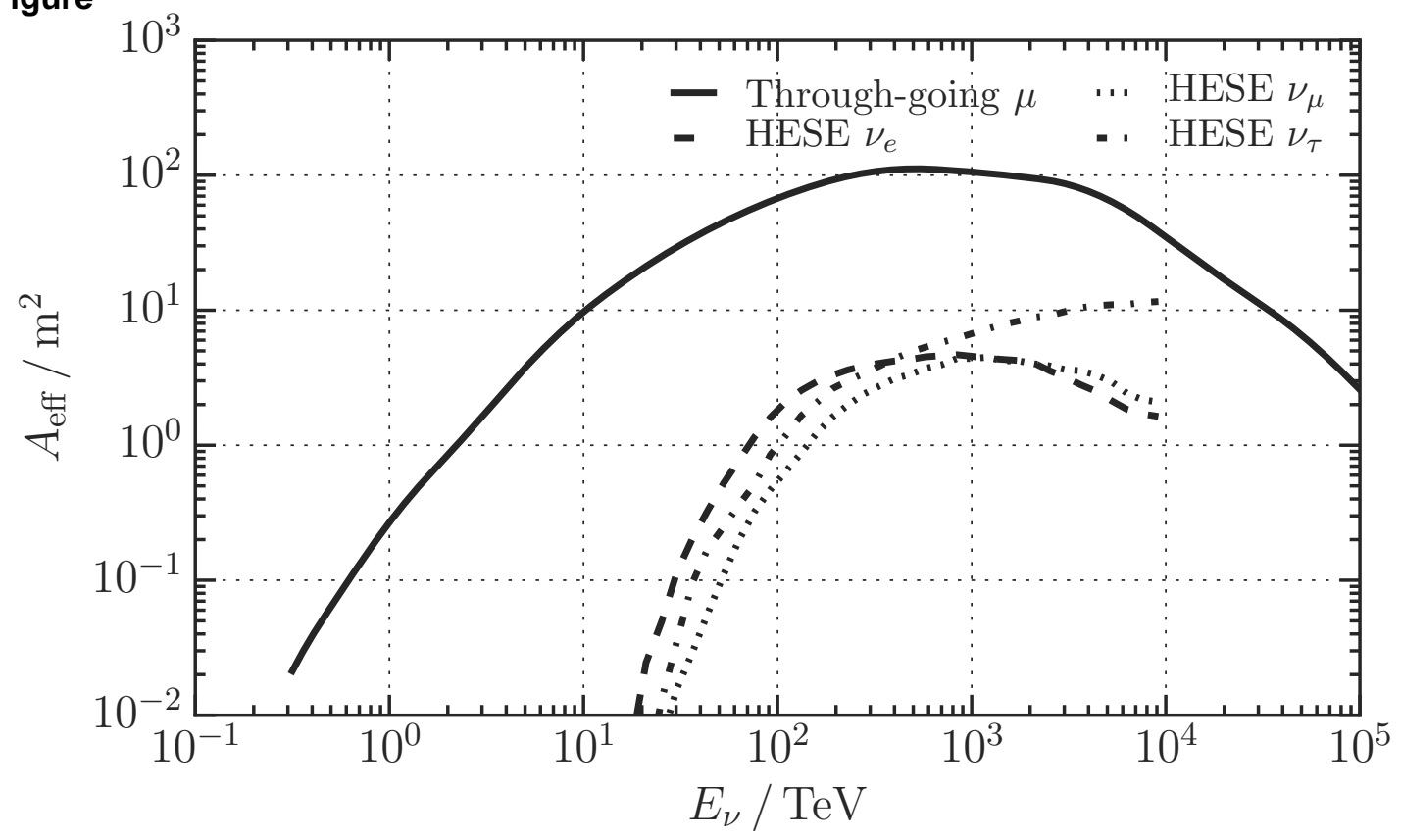




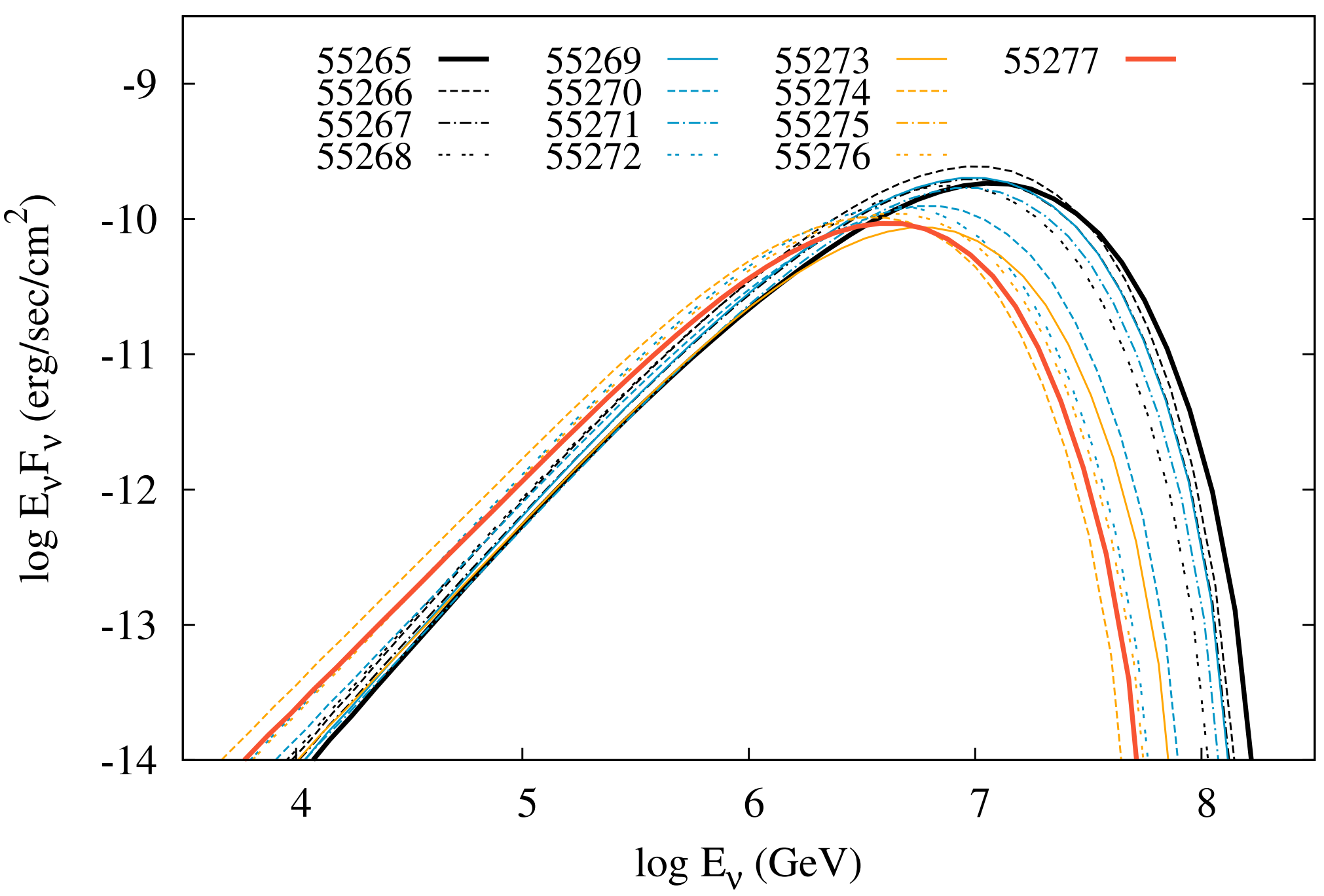

